Article

\title{
A Novel Motion Compensation Scheme for Airborne Very High Resolution SAR
}

\author{
Zhen Chen ${ }^{1}$, Zhimin Zhang ${ }^{2}$, Yashi Zhou ${ }^{1}{ }^{\mathbb{D}}$, Pei Wang ${ }^{2, *}$ and Jinsong Qiu ${ }^{1}$ \\ 1 School of Electronic, Electrical and Communication Engineering, University of Chinese Academy of Sciences, \\ Beijing 100190, China; chenzhen171@mails.ucas.ac.cn (Z.C.); zhouyashi16@mails.ucas.ac.cn (Y.Z.); \\ qiujinsong17@mails.ucas.ac.cn (J.Q.) \\ 2 Department of Space Microwave Remote Sensing System, Aerospace Information Research Institute, \\ Chinese Academy of Sciences, Beijing 100190, China; zmzhang@mail.ie.ac.cn \\ * Correspondence: wangpei@aircas.ac.cn
}

Citation: Chen, Z.; Zhang, Z.; Zhou, Y.; Wang, P. ; Qiu, J. A Novel Motion Compensation Scheme for Airborne Very High Resolution SAR. Remote Sens. 2021, 13, 2729. https://doi.org/10.3390/rs13142729

Academic Editor: Alin Achim

Received: 8 May 2021

Accepted: 8 July 2021

Published: 12 July 2021

Publisher's Note: MDPI stays neutral with regard to jurisdictional claims in published maps and institutional affiliations.

Copyright: (c) 2021 by the authors. Licensee MDPI, Basel, Switzerland. This article is an open access article distributed under the terms and conditions of the Creative Commons Attribution (CC BY) license (https:/ / creativecommons.org/licenses/by/ $4.0 /)$.

\begin{abstract}
Due to the atmospheric turbulence, the motion trajectory of airborne very high resolution (VHR) synthetic aperture radars (SARs) is inevitably affected, which introduces range-variant range cell migration (RCM) and aperture-dependent azimuth phase error (APE). Both types of errors consequently result in defocused images, as residual range- and aperture-dependent motion errors are significant in VHR-SAR images. Nevertheless, little work has been devoted to the range-variant RCM auto-correction and aperture-dependent APE auto-correction. In this paper, a precise motion compensation (MoCo) scheme for airborne VHR-SAR is studied. In the proposed scheme, the motion error is obtained from inertial measurement unit and SAR data, and compensated for with respect to both range and aperture. The proposed MoCo scheme compensates for the motion error without space-invariant approximation. Simulations and experimental data from an airborne 3.6 GHz bandwidth SAR are employed to demonstrate the validity and effectiveness of the proposed MoCo scheme.
\end{abstract}

Keywords: very high resolution SAR; motion compensation; range-variant RCM auto-correction; aperture-dependent phase gradient autofocus

\section{Introduction}

The quivering path of the airborne SAR caused by atmospheric turbulence results in a considerably blurry image, especially for very high resolution (VHR) SAR images. Traditional motion compensation (MoCo) methods for VHR-SAR are burdened by the nonnegligible errors [1]. In this study, a novel MoCo scheme including two modified IMUbased MoCo methods and two improved auto-focus methods is proposed to obtain a wellfocused VHR-SAR image for the stripmap mode by compensating offboard. Inertial measurement units (IMUs) are commonly installed on aircrafts for recording the flight trajectory. Several IMU-based motion compensation (MoCo) methods have been proposed i.e., Two Step MoCo (TSM) [2,3] and One-Step MoCo (OSM) [4,5]. However, the Two Step MoCo only compensates for the first-order phase error before imaging. The compensation is inadequate for VHR-SAR [1,6]. The One-Step MoCo compensates for the range-invariant data displacement and range-variant phase error simultaneously before imaging. It avoids the uncompensated residual range-variant phase error causing the diffusion in azimuth during the range cell migration correction (RCMC). Nevertheless, an underlying assumption is that the range displacement, also called light-of-sight (LOS) data displacement, is invariant with range. The analysis, presented in Section 2, indicates that this assumption results in a residual range-variant data displacement, which cannot be ignored in airborne VHR-SAR imaging.

Several methods (e.g., the chirp scaling [7]) have been proposed to compensate for the residual range-variant data displacement. One is the linear residual displace- 
ment compensation [8]. Nevertheless, it is not sufficient to meet VHR-SAR accuracy requirements. Another possible approach is to divide the wide swath into several subswaths. Then, an identical displacement can be compensated for in each sub-swath $[9,10]$. As the analysis in Section 2 indicates, the precise MoCo requires many sub-swaths, and joining the sub-swaths is inefficient. In this paper, the precise range resampling is employed in the proposed MoCo scheme to compensate for this error without approximation.

Similar to the range-variant motion error, the aperture-dependent motion error is significant when the synthetic aperture is long. The sub-aperture MoCo [11] is commonly employed to compensate for the aperture-dependent motion error. The traditional subaperture MoCo schemes are implemented after RCMC, but the residual aperture-dependent motion error has already been diffused during the RCMC process. In this paper, an improved sub-aperture MoCo implementation is proposed for compensating the residual aperture-dependent motion error precisely before imaging.

As the system bandwidth increases, the resolution becomes finer and IMU records may be not be accurate enough for the VHR-SAR imaging [12-14]. In practice, the IMU-based MoCo alone is usually not sufficient to obtain a well-focused SAR image [15], as the residual uncompensated motion error needs to be estimated from SAR data. The residual range motion error will also cause azimuth phase error (APE). As a result, the point targets far from the range center will be defocused in both the range and azimuth directions. This should, therefore, be considered as a two-dimensional auto-focus problem, which means that azimuth-based autofocus methods like the Phase Gradient Autofocus (PGA) [16,17] can not estimate and compensate for this error precisely.

There are three possible ways to solve the two-dimensional auto-focus problem. First, one can estimate the two-dimensional residual error directly. In [18], the author minimized the entropy of the image in two dimensions by the Newton-Raphson method. The twodimensional iterations are time-consuming. The second way to solve the problem is to estimate and correct the range motion error using azimuth phase correction. In [19-21], the authors focused the image by establishing the relation between the APE and the range motion error. This method cannot compensate for the residual range displacement with sufficient precision for the VHR-SAR imaging. Third, the range-compressed data can be aligned in adjacent pulses using cross-correlation or minimum entropy [22,23]. It is precise and efficient when compensating for the residual range-invariant motion error [24-26]. Nevertheless, the residual range-variant motion error could still be significant in VHR-SAR because little work has been devoted to compensate for it. In this study a range-variant RCM auto-correction method is proposed. Two high signal-to-clutter ratio (SCR) areas are automatically selected from the whole swath for obtaining a reliable estimate of the residual range-variant motion error. The residual range-variant motion error is estimated and determined through a closed-form solution to realize computational efficiency.

Since the IMU is not accurate enough and RCM auto-correction cannot compensate for high-order azimuth phase errors, the APE will exist. Hence, the PGA is still required. However, little work has been devoted to removing the residual video phase (RVP) error in the PGA. As the PGA ignores the aperture-dependent RVP error and deramps the image at the azimuth center, which will reduce the APE estimation accuracy. In this paper, a coarse image is employed to determine the precise azimuth time of the strong points selected for estimating the APE. Thus, the APE can be estimated precisely without RVP error. This operation is implemented at the beginning of the proposed PGA.

\section{Methods}

The geometric relation of an airborne VHR-SAR and the illuminated area is shown in Figure 1. The SAR platform flies along the $y$-axis looking towards its right side at an altitude of $H$. $C, D$, and $E$ are the points on the light-of-sight direction when the aircraft is at $A . C^{\prime}, D^{\prime}, E^{\prime}$ are the corresponding points when the plane is at point $A^{\prime}$ that match the relations of $A C=A^{\prime} C^{\prime}, A D=A^{\prime} D^{\prime}$ and $A E=A^{\prime} E^{\prime}$. The ideal position of the aircraft 
at this azimuth time should be $B^{\prime \prime}$. We assume that a linear frequency-modulated signal is transmitted. Then the received signal can be expressed as

$$
\begin{aligned}
& s_{0}(\tau, \eta)=\omega_{r}\left(\tau-2 R_{s}(\tau, \eta) / c\right) \omega_{a}\left(\eta-\eta_{c}\right) \\
& \exp \left(j \pi K_{\tau}\left(\tau-2 R_{s}(\tau, \eta) / c\right)^{2}\right) \exp \left(-j 4 \pi f_{0} R_{s}(\tau, \eta) / c\right)
\end{aligned}
$$

where the meanings of the notations are listed in the following Table 1.

Table 1. Represented parameters of the notations.

\begin{tabular}{cc}
\hline Notation & Parameters \\
\hline$s_{0}$ & Raw echo data \\
$\tau$ & Fast time \\
$\eta$ & Slow time \\
$\eta_{c}$ & Slow time center \\
$\omega_{r}$ & Range envwlope \\
$\omega_{a}$ & Azimuth envelope \\
$R_{s}$ & Sampling slant range \\
$c$ & Speed of light \\
$K_{\tau}$ & Chirp rate \\
$f_{0}$ & Carrier frequency \\
\hline
\end{tabular}

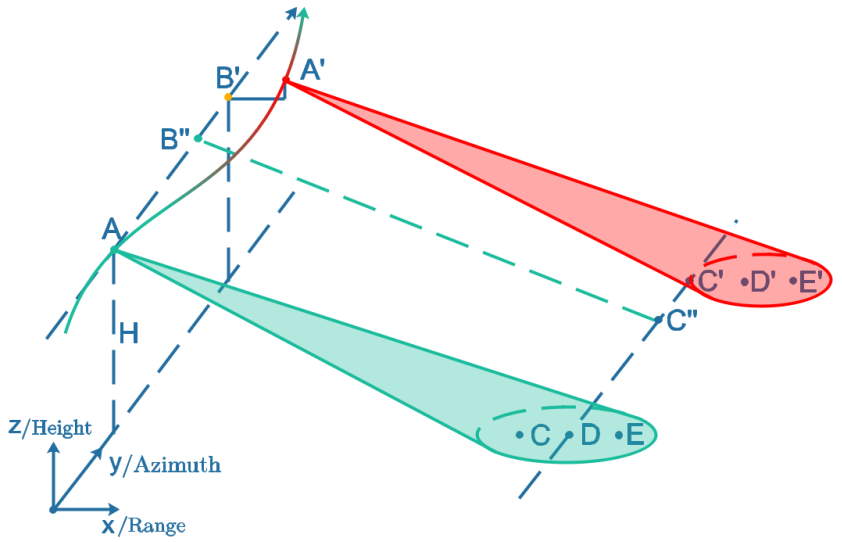

Figure 1. The geometry of the airborne VHR-SAR and its illuminating area.

For accurate range-variant IMU-based MoCo, range compression is applied first. The signal after compression is denoted as $s_{1}(\tau, \eta)$. For a given pulse echo, i.e., when $\eta=\eta_{A^{\prime}}$, the expression for the compressed signal can be expressed as

$$
s_{1}\left(\tau, \eta_{A^{\prime}}\right)=\operatorname{sinc}\left(\tau-2 R_{s}\left(\tau, \eta_{A^{\prime}}\right) / c\right) \exp \left(-j 4 \pi f_{0} R_{S}\left(\tau, \eta_{A^{\prime}}\right) / c\right) .
$$

\subsection{Range-Variant One-Step MoCo}

The LOS motion error is the difference of the sampling slant range and the corresponding ideal slant range. For a given point target, i.e., point $C^{\prime}$ in Figure 1, the motion error is

$$
\Delta R_{C^{\prime}}\left(\tau_{C^{\prime}}, \eta_{A^{\prime}}\right)=R_{A^{\prime} C^{\prime}}-R_{B^{\prime} C^{\prime}}
$$

where $R_{A^{\prime} C^{\prime}}$ and $R_{B^{\prime} C^{\prime}}$ represent the slant ranges $A^{\prime} C^{\prime}$ and $B^{\prime} C^{\prime}$, respectively.

The sampling slant range depends on the sampling delay, which is independent from the motion error. The motion error changes the data locations rather than the slant range itself. Thus sampling slant range can be expressed as $R_{c}+\tau c / 2$, where $R_{c}$ denotes the slant range of the scene's center. The ideal slant range should be calculated together with the sampling slante range and the motion error from IMU records. Then, to align the data with respect to azimuth, the movement or resample in range direction should be done. As shown in Figure 1, the motion error at azimuth time $\eta_{A^{\prime}}$ can be calculated as 


$$
\Delta R\left(\tau, \eta_{A^{\prime}}\right)=R_{c}+\tau c / 2-\sqrt{\left(\sqrt{\left(R_{c}+\tau c / 2\right)^{2}-\left(H+\Delta z\left(\eta_{A}^{\prime}\right)\right)^{2}}+\Delta x\left(\eta_{A}^{\prime}\right)\right)^{2}+H^{2}}
$$

where $\Delta x(\eta)$ and $\Delta z(\eta)$ are the motion errors in the $x$-axis and the $z$-axis directions, respectively.

Due to the motion error, the phase that relying on the range also contains error. In the proposed scheme, the first step is to compensate for the phase error according to the motion error Equation (4). The signal after phase error compensation can be expressed as

$$
\begin{aligned}
s_{2}\left(\tau, \eta_{A^{\prime}}\right) & =s_{1}\left(\tau, \eta_{A^{\prime}}\right) \exp \left(j 4 \pi f_{0} \Delta R\left(\tau, \eta_{A^{\prime}} / c\right)\right) \\
& =\operatorname{sinc}\left(\tau-2 R_{S}\left(\tau, \eta_{A^{\prime}}\right) / c\right) \exp \left(-j 4 \pi f_{0} R_{L O S}\left(\tau, \eta_{A^{\prime}}\right) / c\right)
\end{aligned}
$$

where $R_{L O S}(\tau, \eta)=R_{S}(\tau, \eta)-\Delta R(\tau, \eta)$ represents the slant range after phase error compensation.

Then the correction of data displacement is improved in the scheme since the traditional OSM method is not accurate enough. OSM method multiplies a linear phase term in range frequency domain for correcting the displacement. It compensate the whole swath with the same motion error $\Delta R\left(\tau_{c}, \eta_{A^{\prime}}\right)$. The signal after compensation is

$$
\begin{aligned}
s_{3}\left(\tau, \eta_{A^{\prime}}\right) & =\mathcal{I F}_{r}\left\{\mathcal{F} \mathcal{T}_{r}\left\{s_{2}\left(\tau, \eta_{A^{\prime}}\right)\right\} \exp \left(j 4 \pi f_{r} \Delta R\left(\tau_{C^{\prime}}, \eta_{A^{\prime}}\right) / c\right)\right\} \\
& =\operatorname{sinc}\left(\tau-2\left(R_{s}(\tau, \eta)-\Delta R\left(\tau_{C^{\prime}}, \eta_{A^{\prime}}\right) / c\right) \exp \left(-j 4 \pi f_{0} R_{L O S}(\tau, \eta) / c\right)\right.
\end{aligned}
$$

where $\mathcal{F} \mathcal{T}_{r}$ and $\mathcal{I F}_{r}$ denote the forward and inverse Fourier transforms in range, respectively.

The residual displacement after OSM compensation can be expressed as

$$
\epsilon_{\Delta R}\left(\tau, \eta_{A^{\prime}}\right)=\Delta R\left(\tau, \eta_{A^{\prime}}\right)-\Delta R\left(\tau_{c}, \eta_{A^{\prime}}\right) .
$$

The displacement error $\Delta R\left(\tau, \eta_{A^{\prime}}\right)$ and the residual displacement error $\epsilon_{\Delta R}\left(\tau, \eta_{A^{\prime}}\right)$ for $\Delta x=0.2 \mathrm{~m}, \Delta z=-0.05 \mathrm{~m}$ and a looking angle of $35^{\circ}$ are shown in Figure $2 \mathrm{a}, \mathrm{b}$ respectively. As shown in Figure 2, the residual displacement error exceeds half a range cell when the range offset is larger than $500 \mathrm{~m}$ in $4400 \mathrm{MHz}$ sampling frequency. This indicates that a notable error will occur when using the OSM on VHR-SAR data. Therefore, sinc interpolation is employed in the proposed MoCo scheme for improving the displacement compensation, in which case the compensated data can be expressed as

$$
\begin{aligned}
s_{3}\left(\tau, \eta_{A^{\prime}}\right) & =\sum_{l}\left\{\operatorname{sinc}\left(l-2 \Delta R\left(\tau, \eta_{A^{\prime}}\right) F_{r} / c\right) s_{2}\left(\tau+l / F_{r}, \eta_{A^{\prime}}\right)\right\} \\
& =\operatorname{sinc}\left(\tau-2 R_{L O S}(\tau, \eta) / c\right) \exp \left(-j 4 \pi f_{0} R_{L O S}(\tau, \eta) / c\right)
\end{aligned}
$$

where $F_{r}$ represents the sampling frequency.

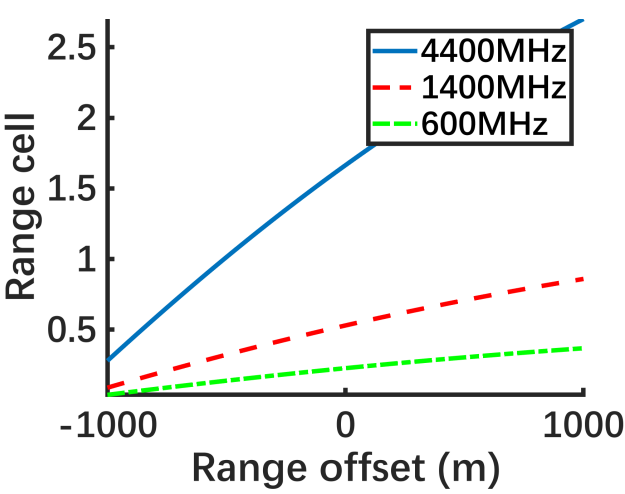

(a)

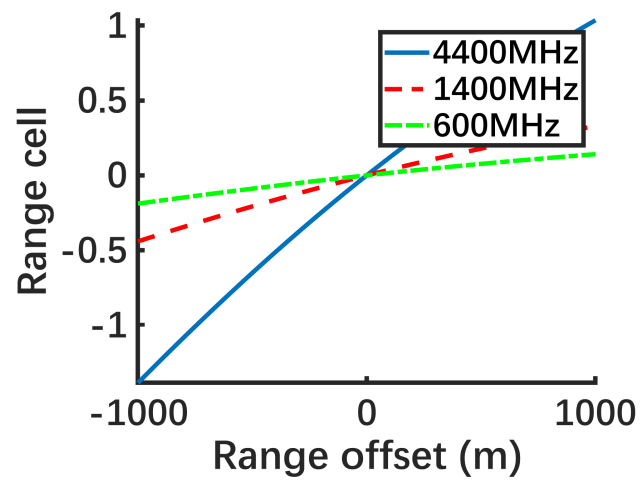

(b)

Figure 2. Instances of the LOS displacement and residual LOS displacement with traditional OSM (a) LOS displacement (b) residual LOS displacement. 


\subsection{Azimuth Resampling and Improved Sub-Aperture MoCo}

In difference to LOS motion error, azimuth motion error varies with azimuth time rapidly. Therefore, its compensation commonly requires accurate resampling in time domain unless the azimuth motion error is simple [27]. Therefore, the proposed scheme use Sinc interpolation to resample the azimuth data. The azimuth resampling can ensure the pulse echoes are uniformly-spaced in azimuth, which is shown in Figure 3. The compensated signal can be expressed as

$$
\begin{aligned}
s_{4}(\tau, \eta) & =\sum_{l}\left\{\operatorname{sinc}\left(l-\Delta y(\eta) F_{a} / V_{r}\right) s_{3}(\tau, \eta)\right\} \\
& =\operatorname{sinc}\left(\tau-2 R_{a r s}(\tau, \eta) / c\right) w_{a}\left(\eta-\eta_{c}\right) \exp \left(-j 4 \pi f_{0} R_{\text {ars }}(\tau, \eta) / c\right)
\end{aligned}
$$

where $R_{\text {ars }}(\tau, \eta)=R_{L O S}\left(\tau, \eta+\Delta y(\eta) / V_{r}\right)$ denotes the slant range after azimuth resampling. $F_{a}$ and $\Delta y(\eta)$ represent the pulse repetition frequency (PRF) and the motion error along the $y$-axis respectively. $V_{r}$ is the equivalent velocity.

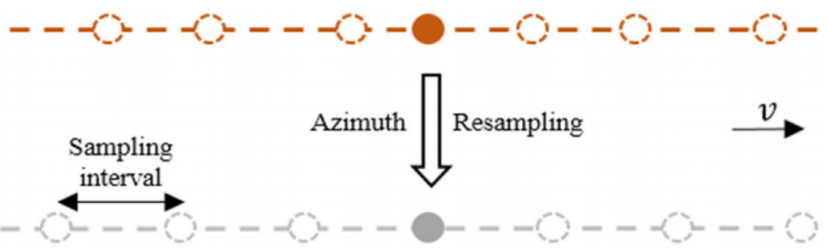

Figure 3. The azimuth resampling diagram.

The LOS motion compensation is implemented before RCMC, therefore each pulse data contain not only LOS echo but also echo from the whole aperture direction. Therefore, the data still retain the residual aperture-dependent motion error [28]. Therefore the Subaperture $\mathrm{MoCo}$ is required to compensate the non-zero Doppler echo data since these data have been compensated with the zero-Doppler motion error. Traditional sub-aperture MoCo [29] compensates this error after RCMC before azimuth compression. The uncompensated aperture-dependent motion error will be diffused during RCMC. To precisely compensate the aperture-dependent motion error, an improved sub-aperture MoCo application before RCMC is integrated in the proposed MoCo scheme. As shown in Figure 4, the echo signal of point $D^{\prime \prime}$ becomes the curve $\widehat{D^{\prime} D^{\prime \prime}}$ after range compression. Point $D^{\prime \prime}$ is sampled in the same range cell to point $D^{\prime}$ at azimuth time $\eta_{A^{\prime}}$ since their sampling delays are equal, i.e., $A^{\prime} D^{\prime \prime}=A^{\prime} D^{\prime}$.

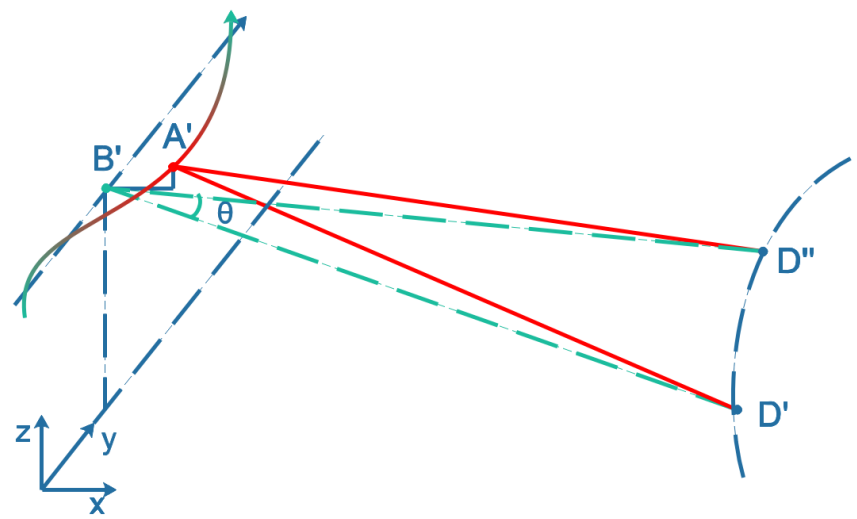

Figure 4. Aperture-dependent motion error.

Therefore, in the precious LOS motion error, point $D^{\prime \prime}$ has compensated for in the same manner as point $D^{\prime}$ by

$$
\Delta R_{D^{\prime}}\left(\tau_{D^{\prime}}, \eta_{A^{\prime}}\right)=R_{A^{\prime} D^{\prime}}-R_{B^{\prime} D^{\prime}} .
$$


Thus, the residual aperture-dependent motion error for point $D^{\prime \prime}$ is

$$
\begin{aligned}
\Delta R_{D^{\prime \prime}}\left(\tau_{D^{\prime}}, \eta_{A^{\prime}}\right)-\Delta R_{D^{\prime}}\left(\tau_{D^{\prime}}, \eta_{A^{\prime}}\right) & =\left(R_{A^{\prime} D^{\prime \prime}}-R_{B^{\prime} D^{\prime \prime}}\right)-\left(R_{A^{\prime} D^{\prime}}-R_{B^{\prime} D^{\prime}}\right) \\
& =R_{B^{\prime} D^{\prime}}-R_{B^{\prime} D^{\prime \prime}} .
\end{aligned}
$$

To compensate the aperture-variant motion error, the SAR data should be separated by aperture angles. By utilizing the equivalent relation between the spatial and frequency domain of azimuth $f_{\eta}(\theta)=2 V_{r} \sin (\theta) / \lambda$, the signal from a narrow sub-aperture can be obtained as

$$
s_{\theta}(\tau, \theta)=\operatorname{rect}\left[f_{\eta}(\theta) / F_{a}\right] \mathcal{F} \mathcal{T}_{a}\left\{s_{4}(\tau, \eta)\right\}
$$

where $\mathcal{F} \mathcal{T}_{a}$ denotes the Fourier transform in azimuth. $\theta$ represents the squint angle of a given sub-sperture.

Then, the aperture-dependent motion error can be compensated for at each angle

$$
\begin{aligned}
s_{5}(\tau, \eta) & =\mathcal{I F}_{a}\left\{\sum_{\theta} s_{\theta}(\tau, \theta) \exp \left(j 4 \pi f_{0}\left(\Delta R_{\theta}(\tau, \eta)-\Delta R(\tau, \eta) / c\right)\right\}\right. \\
& =\operatorname{sinc}\left(\tau-2 R_{\text {sam }}(\tau, \eta) / c\right) w_{a}\left(\eta-\eta_{c}\right) \exp \left(-j 4 \pi f_{0} R_{\text {sam }}(\tau, \eta) / c\right)
\end{aligned}
$$

where $R_{\text {sam }}(\tau, \eta \mid \theta)=R_{\text {ars }}(\tau, \eta \mid \theta)-\Delta R_{\theta}(\tau, \eta)+\Delta R(\tau, \eta)$ represents the slant range after the sub-aperture MoCo. $\mathcal{I} \mathcal{F}_{a}$ denotes the inverse Fourier transform in azimuth.

\subsection{Range-Variant Residual RCM Auto-Correction}

Since the IMU record can not always ensure a final well focusing image. It is necessary to study the motion compensation based on the echo data. In range direction, since the raw data after range compression are skewed and intersect with each other, it is difficult to estimate the residual RCM before RCMC reliably. Therefore, exist RCM auto-correction methods are always implemented after RCMC. Among them, the entropy method, a robust method for estimating the residual RCM [24], is employed and extended in the proposed MoCo scheme. The entropy of the two range profiles is defined as

$$
S_{\eta_{1}, \eta_{2}}\left(\Delta_{r}\right)=-\sum_{n=1}^{N_{e}} p\left(\Delta_{r}, n\right) \ln p\left(\Delta_{r}, n\right)
$$

where

$$
p\left(\Delta_{r}, n\right)=\frac{\left|s_{\eta_{1}}\right|+\left|s_{\eta_{2}+\Delta_{r}}\right|}{\sum_{n=1}^{N_{e}}\left(\left|s_{\eta_{1}}\right|+\left|s_{\eta_{2}+\Delta_{r}}\right|\right)}
$$

$\eta_{1}, \eta_{2}$, and $N_{e}$ denote the two adjacent range profiles and the number of selected range cells, respectively. In traditional residual RCM auto-correction (RRAC), the range profile difference is estimated to be [24]

$$
\hat{\epsilon}_{R}^{\prime}\left(\eta_{2}\right)=\arg \min _{\Delta_{r}} S_{\eta_{1}, \eta_{2}}\left(\Delta_{r}\right)
$$

Aggregating the range profile difference yields the estimated residual $\mathrm{RCM} \epsilon_{R}$

$$
\hat{\epsilon}_{R}(\eta)=\sum_{t_{a}=\eta_{i}}^{\eta} \hat{\epsilon}_{R}^{\prime}\left(t_{a}\right)
$$

where $\eta_{i}$ represents the start time of sub-aperture $i$.

The residual RCM is also varied with range and therefore should be compensated accordingly in VHR-SAR. Therefore, RRAC needs to be extended to range-variant mode. In order to estimate the precise residual RCM for each range bin, we first choose two high SCR areas in the whole swath for the estimation process. In each area, the estimation is 
performed similarly to the traditional method. Suppose the residual RCMs in these two areas are denoted as $\epsilon_{R_{1}}(\eta)$ and $\epsilon_{R_{2}}(\eta)$. Then, they can be obtained as follows.

$$
S_{\eta_{1}, \eta_{2}}^{q}\left(\Delta_{r_{q}}\right)=-\sum_{n=r_{q}-\frac{N_{e}}{2}}^{r_{q}+\frac{N_{e}}{2}-1} p\left(\Delta_{r_{q}}, n\right) \ln p\left(\Delta_{r_{q}}, n\right), q \in\{1,2\}
$$

where

$$
\begin{gathered}
p\left(\Delta_{r_{q}}, n\right)=\frac{\left|s_{\eta_{1}}\right|+\left|s_{\eta_{2}+\Delta_{r_{q}}}\right|}{\sum_{n=r_{q}}^{N_{e}}\left(\left|s_{\eta_{1}}\right|+\left|s_{\eta_{2}+\Delta_{r_{q}}}\right|\right)} \\
\hat{\epsilon}_{R_{q}}^{\prime}\left(\eta_{2}\right)=\arg \min _{\Delta_{r_{q}}} S_{\eta_{1}, \eta_{2}}^{q}\left(\Delta_{r_{q}}\right) \\
\epsilon_{R_{q}}(\eta)=\sum_{t_{a}=\eta_{0}}^{\eta} \hat{\epsilon}_{R_{q}}^{\prime}\left(t_{a}\right)
\end{gathered}
$$

Here $r_{1}$ and $r_{2}$ denote the indices of the range bin of the selected area center, while $N_{e}$ denotes the length of the sliding window.

As shown in Figure 5, the whole azimuth time is divided into several sub-apertures. The high SCR area selection can be chosen automatically using the sliding window method

$$
\begin{gathered}
s_{a c c}(\tau)=\sum_{r=1}^{\tau} \sum_{\eta=\eta_{i}}^{\eta=\eta_{i+1}-1}\left|s_{3}(r, \eta)\right| \\
r_{q}=\arg \max _{r}\left[s_{a c c}(r)-s_{a c c}\left(r-N_{e}\right)\right]-1+\frac{N_{e}}{2} .
\end{gathered}
$$

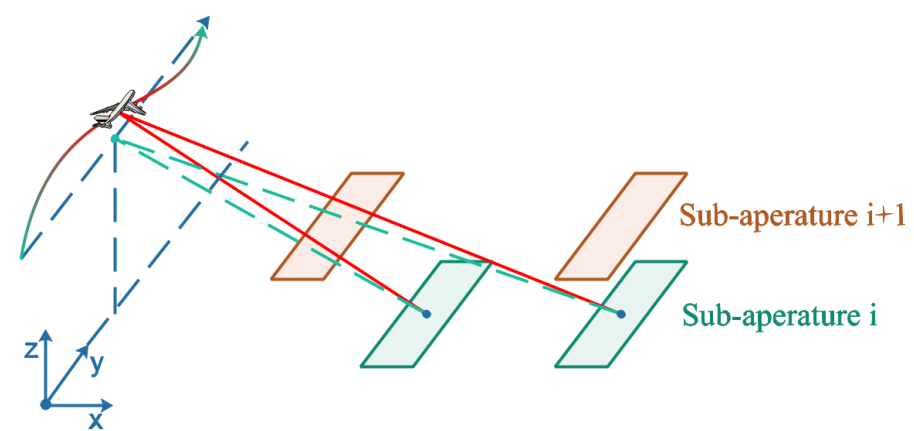

Figure 5. Range-variant residual RCM auto-correction.

Then, the residual range-variant motion error in the whole range swath can be obtained from the estimated residual RCMs. Different from traditional RRAC methods, the estimated range-variant residual RCM is obtained as $\Delta x$ and $\Delta z$ instead of a single movement value, and therefore it should be compensated for by reusing the IMU-based LOS MoCo. The relationships between the estimated residual RCMs and residuals $\Delta x$, and $\Delta z$ can be expressed as

$$
\begin{aligned}
& \epsilon_{R_{1}}(\eta)=R_{1}-\sqrt{\left(\sqrt{R_{1}^{2}-(H+\Delta z(\eta))^{2}}+\Delta x(\eta)\right)^{2}+H^{2}} \\
& \epsilon_{R_{2}}(\eta)=R_{2}-\sqrt{\left(\sqrt{R_{2}^{2}-(H+\Delta z(\eta))^{2}}+\Delta x(\eta)\right)^{2}+H^{2}}
\end{aligned}
$$

where $R_{1}$ and $R_{2}$ represent the real slant range from $r_{1}$ and $r_{2}$ to the aircraft, respectively.

Since the Equation (24) are non-linear, a closed-form solution is deduced. As shown in Figure 6, point $P$ denotes the aircraft, and $X_{1}(\eta)$ and $X_{2}(\eta)$ are the coordinate values of the selected area centers. Then, we have $R_{1}=P X_{1}$ and $R_{2}=P X_{2}$. Since $R_{1}$ and $R_{2}$ are the sampling ranges, their lengths are invariant with azimuth time. $\hat{\epsilon}_{R_{1}}(\eta)$ and $\hat{\epsilon}_{R_{2}}(\eta)$ 
have already been obtained as Equation (24). Therefore, the ideal slant ranges of the two selected area centers are $R_{r 1}=R_{1}-\epsilon_{R_{1}}(\eta)$ and $R_{r 2}=R_{2}-\epsilon_{R_{2}}(\eta)$. As shown in Figure 6, draw two arcs $\overparen{P_{1} P_{2}}$ and $\overparen{P_{3} P_{4}}$ whose centers are at $X_{1}(\eta)$ and $X_{2}(\eta)$ and their radii are $R_{r 1}$ and $R_{r 2}$ respectively. Then the intersection point $P^{\prime}$ of these two circles denotes the real position of the aircraft. Via this geometric relationship, we can obtain $x_{1}(\eta), x_{2}(\eta), z_{1}(\eta)$, and $z_{1}(\eta)$ as

$$
\begin{aligned}
& x_{1}(\eta)=\sqrt{\left(R_{1}-\epsilon_{R_{1}}(\eta)\right)^{2}-H^{2}} \\
& x_{2}(\eta)=\sqrt{\left(R_{2}-\epsilon_{R_{2}}(\eta)\right)^{2}-H^{2}} \\
& \left(x(\eta)-x_{1}(\eta)\right)^{2}+z^{2}(\eta)=R_{1}^{2} \\
& \left(x(\eta)-x_{2}(\eta)\right)^{2}+z^{2}(\eta)=R_{2}^{2}
\end{aligned}
$$

$x_{1}(\eta)$ and $x_{2}(\eta)$ can be calculated using the first two equations. Then, the solution can be organized as

$$
\left\{\begin{array}{l}
x=-\left(R_{1}^{\prime 2}-R_{2}^{\prime 2}-x_{1}(\eta)^{2}+x_{2}(\eta)^{2}\right) / \\
z= \pm \sqrt{-\left(A^{2}-C^{2}\right)\left(B^{2}-C^{2}\right)} /(2 C)
\end{array}\right.
$$

where

$$
\begin{aligned}
& R_{1}^{\prime}=R_{1}+\epsilon_{R_{1}}(\eta) \\
& R_{2}^{\prime}=R_{2}+\epsilon_{R_{2}}(\eta) \\
& A=R_{1}^{\prime}+R_{2}^{\prime} \\
& B=R_{1}^{\prime}-R_{2}^{\prime} \\
& C=x_{1}(\eta)-x_{2}(\eta) .
\end{aligned}
$$

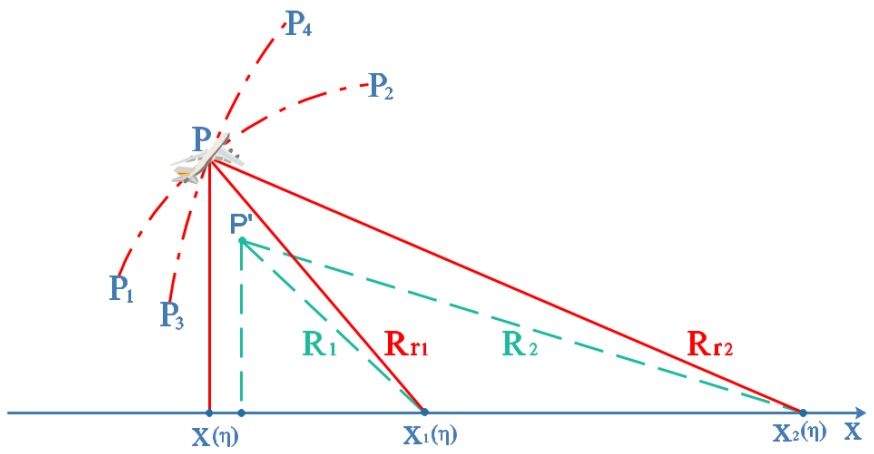

Figure 6. Geometric analysis for deriving the closed-form solution of $\Delta x(\eta)$ and $\Delta z(\eta)$.

Because of symmetry, $z(\eta)$ has two solutions. Since the height of platform is nonnegative, only the positive solution is retained. Subsequently, $\Delta x(\eta)$ and $\Delta z(\eta)$ can be calculated as

$$
\left\{\begin{array}{l}
\Delta x(\eta)=x(\eta) \\
\Delta z(\eta)=|z(\eta)|-H
\end{array}\right.
$$

Then we implement the proposed range-variant RRAC. Suppose the signal after imaging is $s_{6}(\tau, \eta)$. The uncompressed signal can be obtained by applying azimuth decompression to $s_{6}(\tau, \eta)$ as

$$
\begin{aligned}
s_{7}(\tau, \eta) & =\mathcal{I} \mathcal{F}\left\{\mathcal{F} \mathcal{T}\left\{s_{6}(\tau, \eta)\right\} \exp \left(j \pi f_{\eta}^{2} / K a\right)\right\} \\
& =\operatorname{sinc}\left(\tau-2 R_{i m g}(\tau, \eta) / c\right) w_{a}\left(\eta-\eta_{c}\right) \exp \left(-j 4 \pi f_{0} R_{i m g}(\tau, \eta) / c\right)
\end{aligned}
$$

where $R_{\text {img }}$ denotes the slant range after imaging.

Finally, the data after auto-correction can be expressed as

$$
s_{8}(\tau, \eta)=\operatorname{sinc}\left(\tau-2 R_{\text {aut }}(\tau, \eta) / c\right) w_{a}\left(\eta-\eta_{c}\right) \exp \left(-j 4 \pi f_{0} R_{\text {aut }}(\tau, \eta) / c\right)
$$


where $R_{\text {aut }}$ represents the slant range after RCM auto-correction.

During the procedure, a traditional technique for estimating the residual RCM accurately is to upsample the data with respect to range. However, it demands a correspondingly large memory. An alternative and effective way is to utilize identical shifting, as shown in Equation (6), to slide the profile by a given length.

Azimuth downsampling is traditionally employed to ensure computational efficiency. On the one hand, downsampling by a high rate may lead to invalid estimation because of the adjacent coherence loss. Furthermore, it inhibits the detection of high frequency motion errors. The high frequency and low frequency refers to the comparison to the aperture time. If the error is monotonous in an aperture time, it can be considered as a low frequency motion error. On the other hand, the low frequency small motion error will be ignored if there is no downsampling toward azimuth. To handle the problem, in the proposed method, the strategy is enlarging the interval of the adjacent profiles until a non-zero residual RCM is detected.

\subsection{Aperture-Dependent PGA}

After the previous $\mathrm{MoCo}$, the last uncompensated residual motion error is the residual APE. Phase gradient autofocus is commonly employed for compensating the APE. Since RCMC has been implemented, signal $s_{8}$ can be considered as a LFM signal in azimuth attached with the residual APE $\phi(\eta)$

$$
s_{8}(\tau, \eta)=\operatorname{sinc}\left(\tau-2 R_{i}(\tau, \eta) / c\right) w_{a}\left(\eta-\eta_{c}\right) \exp \left(-j \pi K_{\eta}\left(\eta-\eta_{c}\right)^{2}\right) \exp (j \phi(\eta))
$$

where $K_{\eta}$ denotes the chirp rate in azimuth. $\eta_{c}$ represents the center of azimuth time of the point target.

Traditionally, PGA begins in the phase history domain. The azimuth deramping is first applied and the signal becomes

$$
\begin{aligned}
s_{d c}(\tau, \eta) & =s_{8}(\tau, \eta) \exp \left(j \pi K_{\eta} \eta^{2}\right) \\
& =\operatorname{sinc}\left(\tau-2 R_{i}(\tau, \eta) / c\right) w_{a}\left(\eta-\eta_{c}\right) \exp \left(-K_{\eta}\left(\eta_{c}^{2}-2 \eta \eta_{c}\right)\right) \exp (j \phi(\eta))
\end{aligned}
$$

where $K_{\eta}=\left(2 V_{r}^{2}\right) /\left(\lambda R_{0}\right)$.

Traditional PGA deramps the signal at the azimuth center, therefore the estimated APE contains RVP error. The inaccurately estimated phase error will therefore degrade the focus of image. To estimate the APE without RVP, in the proposed scheme, the coarsely focused image before PGA is employed to locate the position of strong points. Then, we can eliminate RVP error by deramping at the locations of different strong point targets. Suppose the strong point position of range bin $\tau$ in sub-aperture $i$ is $y(\tau, i)$, where $\eta_{c}(\tau)=$ $y(\tau, i)\left(V_{r} / F_{a}\right)$. Implementing the exact deramping to $s_{8}(\tau, \eta)$ yields:

$$
\begin{aligned}
s_{d c}\left(\tau^{\prime}, \eta, i\right) & =s_{8}\left(\tau^{\prime}, \eta\right) \exp \left(j \pi K_{\eta}\left(\eta-y\left(\tau^{\prime}, i\right) \frac{V_{r}}{F_{a}}\right)^{2}\right) \\
& =\operatorname{sinc}\left(\tau-2 R_{i}\left(\tau^{\prime}, \eta\right) / c\right) w_{a}(\eta) \exp (j \phi(\eta))
\end{aligned}
$$

where $\tau^{\prime}$ denotes the fast time of the selected range bin.

Then, we can implement the remaining PGA steps and obtain the precise APE estimation. Suppose the data after compensating for the residual APE are $s_{9}(\tau, \eta)$. The final well-focused image $s_{10}(\tau, \eta)$ can be obtained by applying azimuth compression to $s_{9}(\tau, \eta)$. The flowchart of the proposed MoCo scheme is shown in Figure 7. The left side of the figure shows how a point target is focused with the proposed MoCo scheme. 


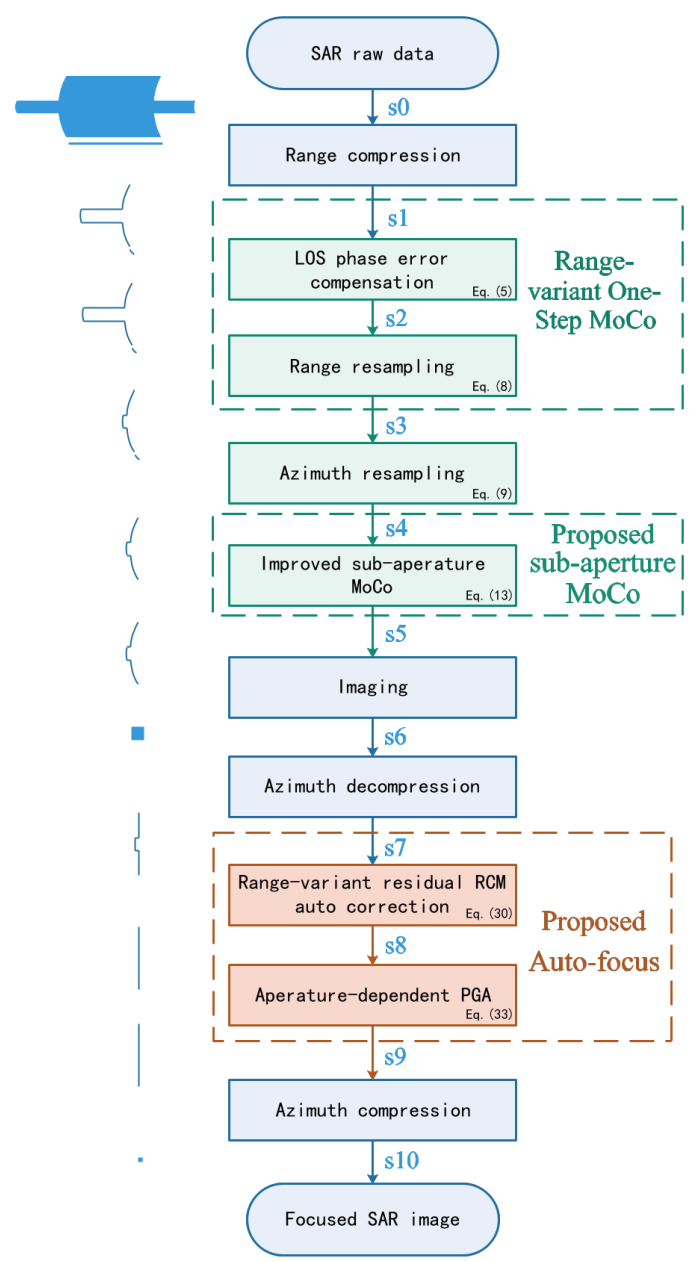

Figure 7. Flowchart of proposed MoCo scheme.

\section{Point Target Simulation Result}

To verify the proposed MoCo scheme, in this section the range-variant OSM (RvOSM), improved sub-aperture MoCo, range-variant RCM auto-correction and aperture-dependent PGA are simulated and analyzed. The parameters for simulations are listed in Table 2 . The imaging area and the point targets are shown in Figure 8. In order to avoid the split in the final point targets introduced by the ladder-shaped frequency spectrum, for the simulation we generated a rectangular spectrum signal by controlling the synthetic aperture angle.

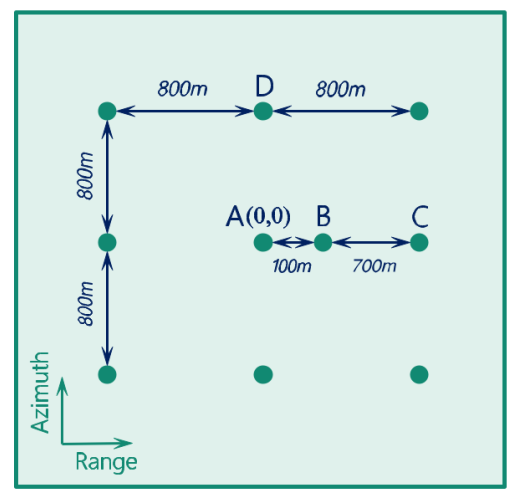

Figure 8. Geometric layout of the points on the ground for simulations. 
Table 2. Parameters in Point Target Simulations.

\begin{tabular}{cc}
\hline Parameter & Value \\
\hline Carrier frequency & $9.6 \mathrm{GHz}$ \\
Velocity & $100 \mathrm{~m} / \mathrm{s}$ \\
Height & $3600 \mathrm{~m}$ \\
Incidence angle & $35 \mathrm{deg}$ \\
Signal bandwidth & $3600 \mathrm{MHz}$ \\
Sampling frequency & $4400 \mathrm{MHz}$ \\
Signal pulse duration & $15 \mu \mathrm{s}$ \\
PRF & $3000 \mathrm{~Hz}$ \\
Length of antenna & $0.496 \mathrm{~m}$ \\
Height of antenna & $0.32 \mathrm{~m}$ \\
\hline
\end{tabular}

\subsection{Range-Variant One-Step MoCo}

To verify the effectiveness of the range-variant OSM, the motion error is added to the simulation echo data as shown in Equation (34). The simulation results are shown in Figure 9, where the traditional OSM is simulated for comparison.

$$
\begin{aligned}
& \Delta x(\eta)=3 c / F_{r} \sin \left(2 \pi \eta / T_{a}\right) \\
& \Delta y(\eta)=3 V_{r} / F_{a} \sin \left(2 \pi \eta / T_{a}+\pi / 4\right) \\
& \Delta z(\eta)=2 c / F_{r} \sin \left(2 \pi \eta / T_{a}+\pi / 2\right)
\end{aligned}
$$

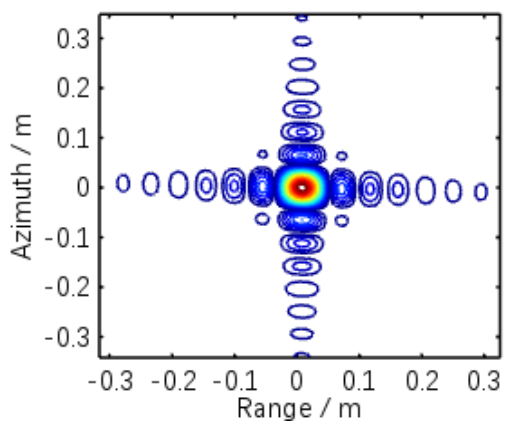

(a)

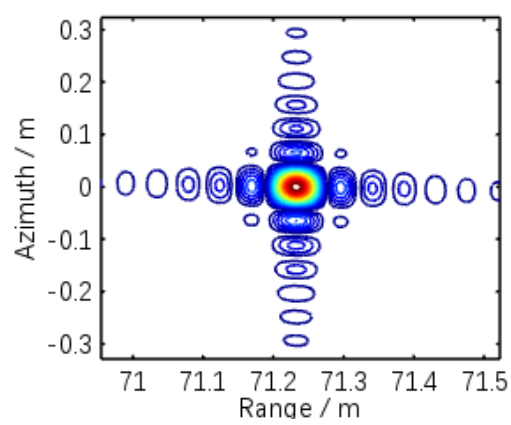

(d)

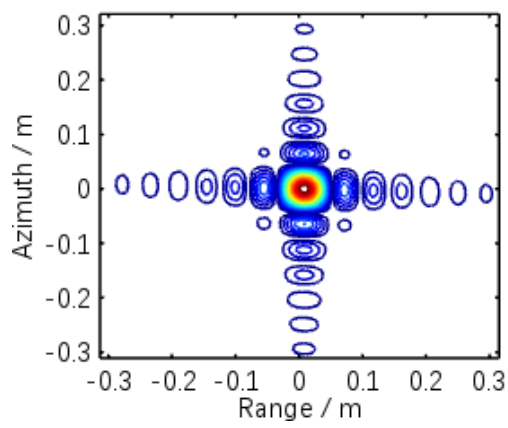

(b)

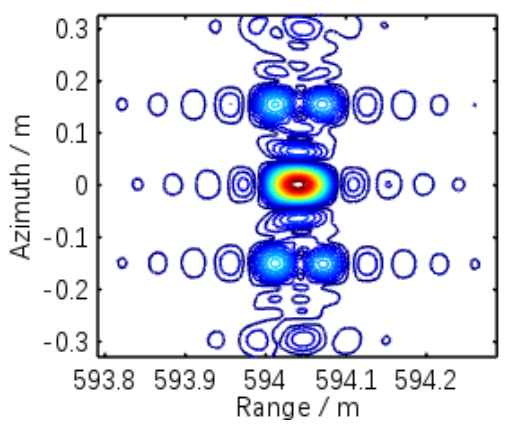

(e)

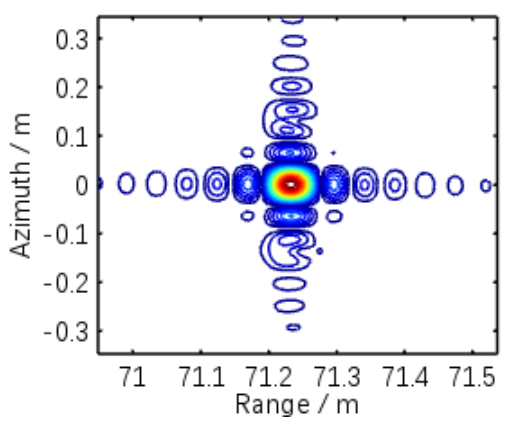

(c)

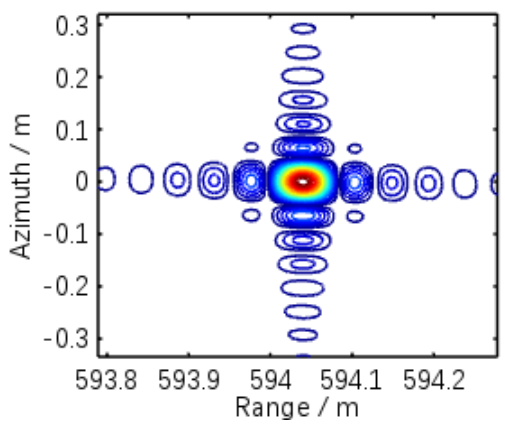

(f)

Figure 9. Comparison results of signal $s_{3}$ with the traditional OSM and RvOSM. (a) Point A with traditional OSM. (b) Point A with RvOSM. (c) Point B with traditional OSM. (d) Point B with RvOSM. (e) Point C with traditional OSM. (f) Point C with RvOSM.

As shown in Figure 9a,c,e, with traditional OSM the residual motion error becomes significant as the point is further away from the range center. In Figure 9e, it is clear that the uncompensated displacement causes point target expanding in range. Since the data are not aligned in a range bin, the compressed point target is not well focused. In Figure $9 b, d, f$, it is clear that the proposed RvOSM, the point targets are well focused. The comparison 
results indicate that the displacement should be precisely compensated in VHR-SAR. The focusing analysis of these point targets are shown in Table 3, where "OSM", "RvOSM" and "Res" represent the traditional One-Step MoCo, the proposed range-variant One-Step MoCo and resolution respectively.

Table 3. Point Target Analysis of One-Step MoCo with Signal $s_{3}$.

\begin{tabular}{cccccccc}
\hline \multirow{2}{*}{ Point } & \multirow{2}{*}{ Method } & \multicolumn{3}{c}{ Azimuth } & \multicolumn{3}{c}{ Range } \\
\cline { 3 - 7 } & & PSLR/dB & ISLR/dB & Res/m & PSLR/dB & ISLR/dB & Res/m \\
\hline A & OSM & -12.77 & -10.09 & 0.249 & -13.27 & -10.22 & 0.042 \\
A & RvOSM & -12.77 & -10.09 & 0.249 & -13.27 & -10.22 & 0.042 \\
B & OSM & -12.49 & -9.960 & 0.249 & -13.40 & -10.50 & 0.042 \\
B & RvOSM & -12.88 & -10.09 & 0.248 & -13.24 & -10.20 & 0.042 \\
C & OSM & -13.14 & -7.252 & 0.244 & 19.70 & -17.17 & 0.049 \\
C & RvOSM & -13.22 & -9.985 & 0.248 & -13.32 & -10.28 & 0.042 \\
\hline
\end{tabular}

\subsection{Improved Sub-Aperture MoCo}

To validate the effectiveness of the improved sub-aperture MoCo, simulations with a large synthetic aperture angle are presented. The length of the antenna was set to $0.1 \mathrm{~m}$. The comparison results are shown in Figure 10. Figure 10a is the imaging result that the aperturedependent residual motion error has been compensated after RCMC with traditional subaperture MoCo. Figure 10b shows the imaging result with the proposed sub-aperture MoCo. This comparison proves the effectiveness of the proposed sub-aperture MoCo. The residual aperture-dependent motion error will be diffused during RCMC, which makes the imaging result not ideal with the traditional sub-aperture MoCo. The proposed method is implemented before RCMC, so all the time- and aperture-variant motion error can be compensated. We can see that the focusing in Figure 10d is further improved compared to Figure 10c. Since the simulation result of Point $A$ is the same as Point $D$, we only present the azimuth upsampling analysis of point D in Figure 11, where "sub-ap." refers to sub-aperture. The detail indices are shown in Table 4, where "Tra" and "Pro" represent the traditional sub-aperture MoCo and the proposed method, respectively. The analysis indicates that the aperture-dependent residual motion error does affect focusing, especially toward azimuth in the VHR-SAR images, and the improved sub-aperture MoCo can compensate for the aperture-dependent residual motion error precisely.

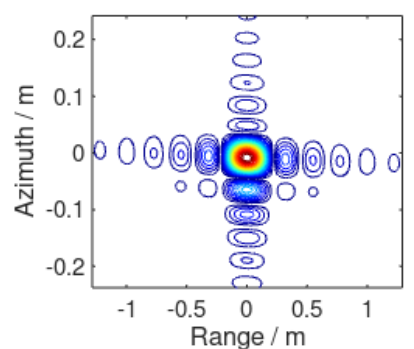

(a)

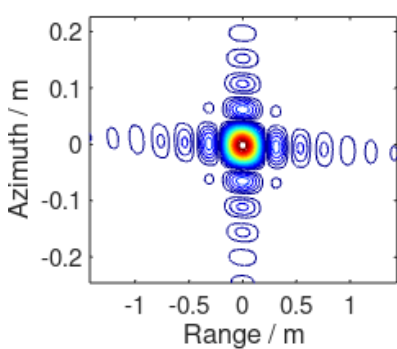

(b)

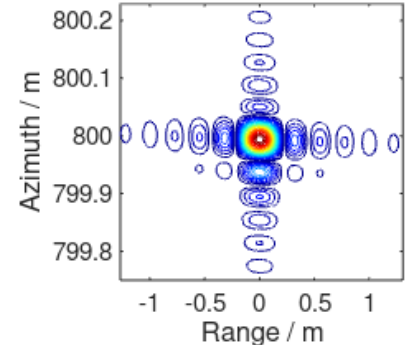

(c)

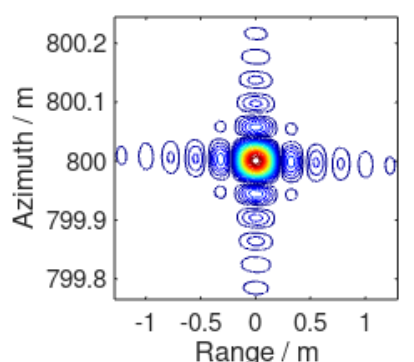

(d)

Figure 10. Comparison results of signal $s_{5}$ with the proposed/traditional sub-aperture MoCo. (a) Imaging result of Point A with traditional sub-aperture MoCo. (b) Point A with proposed sub-aperture MoCo. (c) Point D with traditional sub-aperture MoCo. (d) Point D with proposed sub-aperture MoCo. 


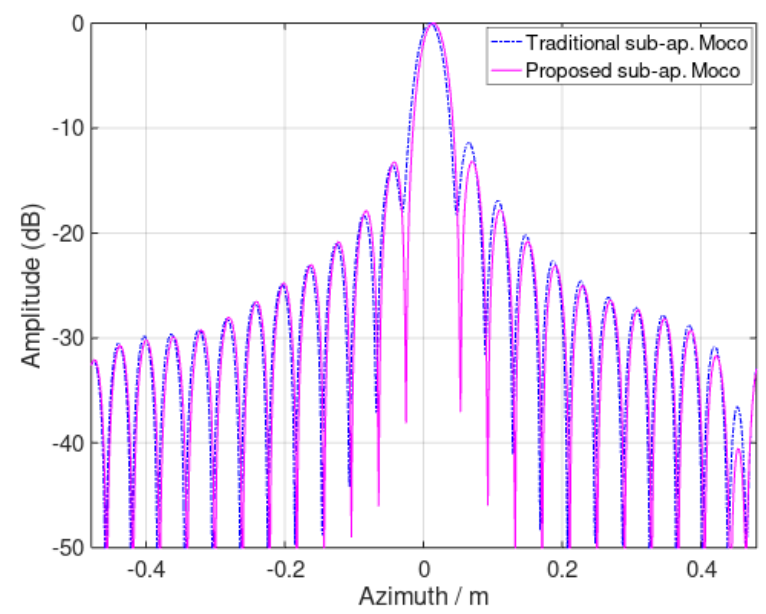

Figure 11. Azimuth upsampling envelope of point D in signal $s_{5}$ with different sub-aperture MoCo methods.

Table 4. Point Target Analysis of Sub-Aperture MoCo with Signal $s_{5}$.

\begin{tabular}{cccccccc}
\hline \multirow{2}{*}{ Point } & \multirow{2}{*}{ Method } & \multicolumn{3}{c}{ Azimuth } & \multicolumn{3}{c}{ Range } \\
\cline { 3 - 7 } & & PSLR/dB & ISLR/dB & Res/m & PSLR/dB & ISLR/dB & Res/m \\
\hline A & Tra & -11.49 & -9.080 & 0.050 & -13.12 & -10.44 & 0.042 \\
A & Pro & -13.23 & -10.08 & 0.050 & -13.15 & -10.62 & 0.042 \\
D & Tra & -11.37 & -9.231 & 0.050 & -13.15 & -10.43 & 0.042 \\
D & Pro & -13.24 & -10.08 & 0.050 & -13.15 & -10.62 & 0.042 \\
\hline
\end{tabular}

\subsection{Range-Variant Residual RCM Auto-Correction}

To verify the effectiveness of the proposed range-variant RRAC (RvRRAC), motion error was added to the echo data as Equations (35)-(37). In order to simulate the case that the IMU data are not precise, IMU-Based MoCo was disabled. To test the robustness of detecting high-frequency motion error together with low-frequency motion error, the added motion error comprises a step function and a slope rise function.

$$
\begin{array}{ll}
\Delta x(\eta)= & \\
0.3, & k T_{e} \leq \eta<\frac{400}{F_{a}}+k T_{e} \\
0.3+0.012\left(\eta-k T_{e}\right), & \frac{400}{F_{a}}+k T_{e} \leq \eta<\frac{500}{F_{a}}+k T_{e} \\
-0.15, & \frac{500}{F_{a}}+k T_{e} \leq \eta<\frac{900}{F_{a}}+k T_{e} \\
-0.15-0.012\left(\eta-k T_{e}\right), & \frac{900}{F_{a}}+k T_{e} \leq \eta<\frac{1000}{F_{a}}+k T_{e}
\end{array}
$$

where $T_{e}=1000 / F_{a}, k \in N$ and $k T_{e} \leq \max (\eta)$.

$$
\begin{gathered}
\Delta y(\eta)=0.1 V_{r} / F_{a} \sin \left(2 \pi \eta / T_{a}+\pi / 4\right) \\
\Delta z(\eta)=-0.025 c / F_{r} \sin \left(2 \pi \eta / T_{a}+\pi / 2\right)
\end{gathered}
$$

As shown in Figure 12a, point B is not well-focused with traditional RRAC. The uncompensated motion error makes the point target echo diffuse across several range bins. The focusing is improved in Figure $12 \mathrm{~b}$ with the proposed RvRRAC. Because the rangevariant residual RCM is significant in VHR-SAR, the residual motion error is spread over several range bins as shown in Figure 13. As shown in Figure 12c, the point target far from the scene center is defocused. By implementing the proposed RvRRAC, the estimated residual RCM is range-variant. As shown in Figure 13, the residual error of the estimated residual RCM is much smaller than that present in a range cell. Besides, we can see that both the high-frequency and low-frequency motion error can be estimated precisely. Then, the residual APE can be compensated using PGA. A well-focused image is obtained in Figure 12d. The focusing analysis metrics are shown in Table 5, where "RRAC" and 
"RvRRAC" represent the residual RCM auto-correction and range-variant RCM autocorrection, respectively.

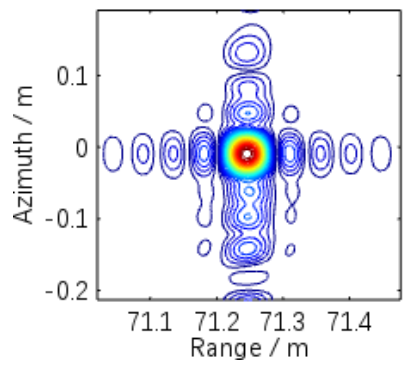

(a)

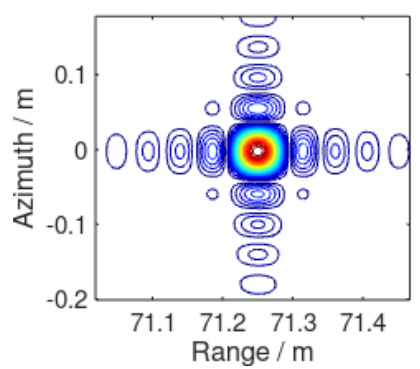

(b)

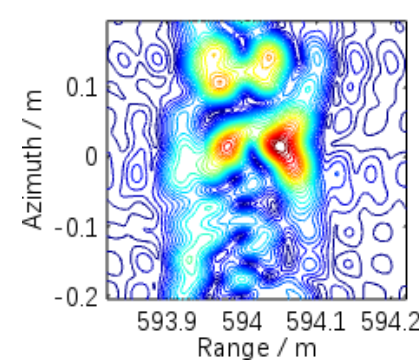

(c)

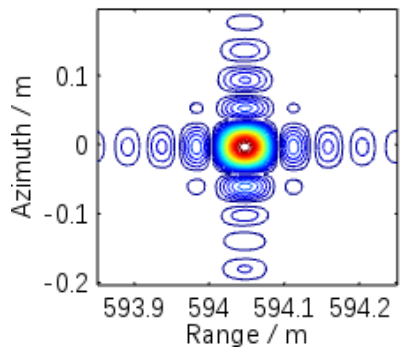

(d)

Figure 12. Comparison results of signal $s_{8}$ with the proposed range-variant/traditional RRAC. (a) The result for point $B$ with traditional RRAC. (b) Point B with the proposed RvRRAC. (c) Point C with traditional RRAC. (d) Point C focused with RvRRAC.

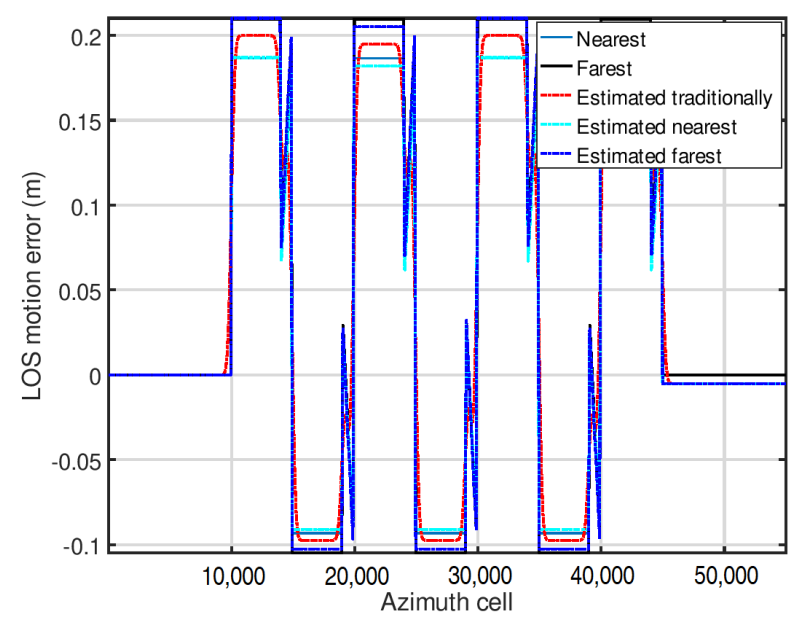

Figure 13. Comparison of added motion error and estimated motion error of Point A in signal $s_{8}$.

Table 5. Point Target Analysis of Residual LOS Motion Error Auto-Correction with Signal $s_{8}$.

\begin{tabular}{cccccccc}
\hline \multirow{2}{*}{ Point } & \multirow{2}{*}{ Method } & \multicolumn{3}{c}{ Azimuth } & \multicolumn{3}{c}{ Range } \\
\cline { 3 - 7 } & & PSLR/dB & ISLR/dB & Res/m & PSLR/dB & ISLR/dB & Res/m \\
\hline B & RRAC & -5.457 & -4.407 & 0.524 & -13.29 & -10.04 & 0.042 \\
B & RvRRAC & -13.10 & -10.01 & 0.249 & -13.29 & -10.10 & 0.042 \\
C & RRAC & -8.018 & -6.432 & 0.436 & -5.053 & -3.245 & 0.050 \\
C & RvRRAC & -13.10 & -9.826 & 0.249 & -13.30 & -10.10 & 0.042 \\
\hline
\end{tabular}

\subsection{Improved PGA Simulation}

In this subsection, the simulation results of traditional PGA and aperture-dependent PGA are compared. A motion error was added to the echo data as described in Equation (38). IMU-Based MoCo was disabled in this simulation.

$$
\Delta y(\eta)=3 \frac{V_{r}}{F_{a}} \sin \left(\frac{2 \pi}{T_{a}} \eta+\frac{\pi}{4}\right)
$$

By implementing traditional PGA, we obtain a focused depiction of Point A, as shown in Figure 14a since the RVP error is not significant in the azimuth center. However it is significant in the areas far from azimuth center. Therefore as shown in Figure 14c, after implementing traditional PGA, Point D still contains RVP error, which degrades its focus. 
As shown in Figure 14d, using the proposed aperture-dependent PGA we can acquire an ideally-focused point target. The analysis of focusing metrics is shown in Table 6, where "AdPGA" represents the proposed aperture-dependent PGA.

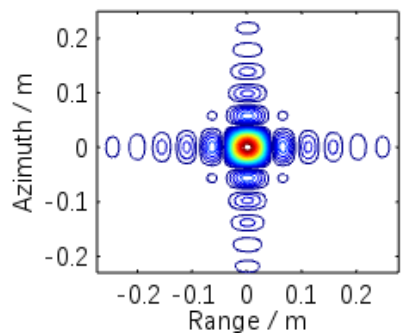

(a)

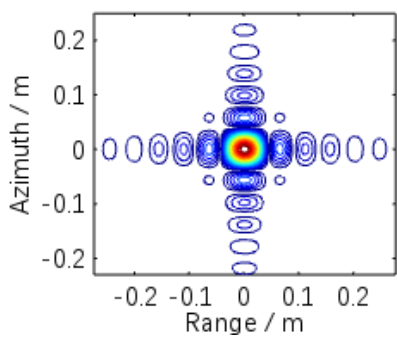

(b)

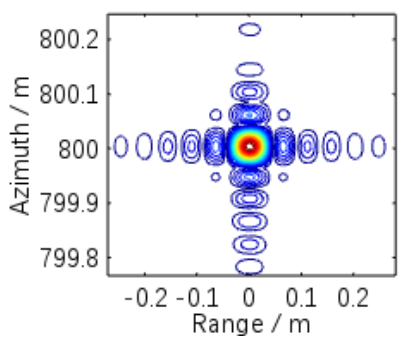

(c)

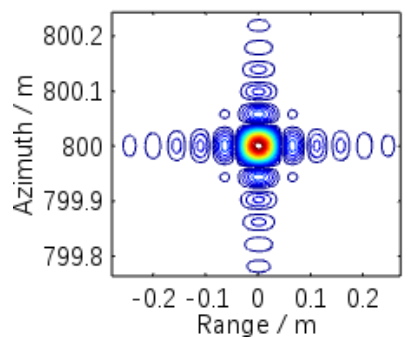

(d)

Figure 14. Comparison results of signal $s_{9}$ with the proposed aperture-dependent and traditional PGA. (a) Point A with traditional PGA. (b) Point A with proposed PGA. (c) Point D with traditional PGA. (d) Point D with proposed PGA.

Table 6. Point Target Analysis of PGA Simulation with Signal s9.

\begin{tabular}{cccccccc}
\hline \multirow{2}{*}{ Point } & \multirow{2}{*}{ Method } & \multicolumn{3}{c}{ Azimuth } & \multicolumn{3}{c}{ Range } \\
\cline { 3 - 7 } & & PSLR/dB & ISLR/dB & Res/m & PSLR/dB & ISLR/dB & Res/m \\
\hline A & PGA & -12.67 & -10.13 & 0.249 & -13.31 & -10.10 & 0.042 \\
A & AdPGA & -12.67 & -10.13 & 0.249 & -13.31 & -10.10 & 0.042 \\
D & PGA & -12.82 & -9.973 & 0.275 & -13.34 & -10.13 & 0.042 \\
D & AdPGA & -13.23 & -10.25 & 0.253 & -13.33 & -10.11 & 0.042 \\
\hline
\end{tabular}

\subsection{The Proposed MoCo Scheme}

To illustrate the effectiveness of the proposed MoCo scheme, the proposed MoCo scheme is compared with the traditional one in this subsection. Figure 15a shows the image obtained with the traditional MoCo techniques. The defocused point targets are distributed on the edges due to the residual range-variant and aperture-dependent motion error. In Figure 15b, the point targets on the edges are well focused since the residual range- and aperture-variant motion error has been compensated by the proposed MoCo scheme precisely.

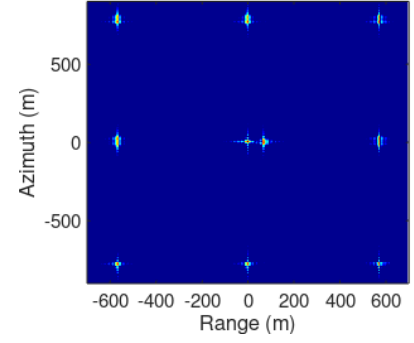

(a)

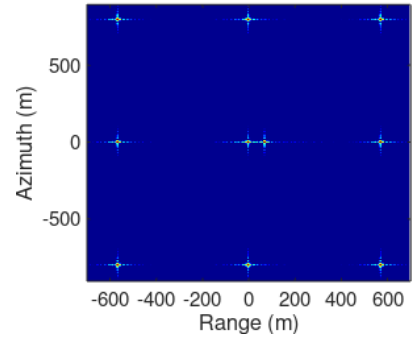

(b)

Figure 15. Comparison results of the proposed MoCo scheme and traditional MoCo techniques with signal s9. (a) Imaging result with traditional MoCo techniques. (b) Imaging result with the proposed MoCo scheme.

Table 7 shows the imaging analysis on point A with different MoCo scheme. where "Tra" and "Pro" denotes the traditional and proposed MoCo scheme respectively. Since the proposed MoCo method focus on improving the range- and azimuth- variant motion error, there is no significant focusing difference in the scene center. 
Table 7. Point Target Analysis of Point A in Signal s $s_{9}$ with Different MoCo Schemes.

\begin{tabular}{cccccccc}
\hline \multirow{2}{*}{ Point } & \multirow{2}{*}{ Method } & \multicolumn{3}{c}{ Azimuth } & \multicolumn{3}{c}{ Range } \\
\cline { 3 - 8 } & & PSLR/dB & ISLR/dB & Res/m & PSLR/dB & ISLR/dB & Res/m \\
\hline A & None & -3.251 & -6.720 & 0.518 & -7.620 & -4.504 & 0.079 \\
A & Tra & -13.20 & -10.10 & 0.251 & -13.28 & -10.13 & 0.042 \\
A & Pro & -13.21 & -10.10 & 0.250 & -13.29 & -10.13 & 0.042 \\
\hline
\end{tabular}

\section{Experimental Results}

In this section, experimental results based on a $3.6 \mathrm{GHz}$-bandwidth SAR are presented to demonstrate the effectiveness of the proposed MoCo scheme. The raw data were collected using an X-band experimental airborne SAR system [30], made by the Institute of Electronics, Chinese Academy of Sciences (IECAS) and designed to prove the functions and parameters of a future spaceborne SAR system. The flight area of the experiment is a flat seaside. Therefore the geometry for imaging can be considered as Figure 1. For the application of the RvRRAC and aperture-dependent PGA, each azimuth sub-aperture was half-overlapped. The flight path of the aircraft is shown in Figure 16.

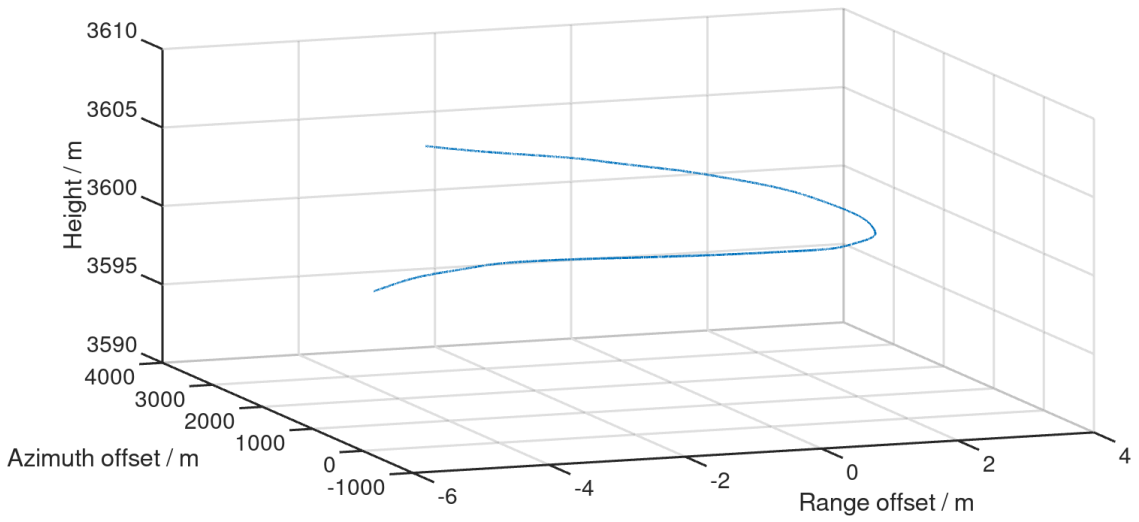

Figure 16. The flighting path.

The final SAR images processed with the TSM, traditional OSM, and proposed MoCo schemes are shown in Figure 17a-c, respectively. The swath of the imaging data is $6989.4 \mathrm{~m}$ (slant range) $\times 3522.2 \mathrm{~m}$ (azimuth). Areas A was selected for detailing the focus comparison since it contained strong points. Figure $18 \mathrm{a}, \mathrm{d}, \mathrm{g}$, are the enlarged images of Aeras A. It is a shed house image. From Figure $18 \mathrm{~b}, \mathrm{e}, \mathrm{h}$, we can see the arranged screws. They are the enlarged images of Aeras B. Figure 18c,f,i, are the enlarged images of Aera C. They are the upsampling slices of three screws. From thses figures, we can see that the screws are defocused slightly in Figure 18b,c toward range direction, which indicates the range-variant phase error is significant and should be compensated before RCMC. The screws are also defocused in Figure 18e,f in azimuth direction, which indicates that the range-variant displacement error is also significant and should be compensated. Notice that the range offset is slightly changed from Figure $18 \mathrm{c}, \mathrm{f}, \mathrm{i}$. This phenomenon indicates the range displacement has been compensated. Thus the residual APE error can be auto compensated by PGA. The system parameters of this SAR are those presented in Table 8 . 


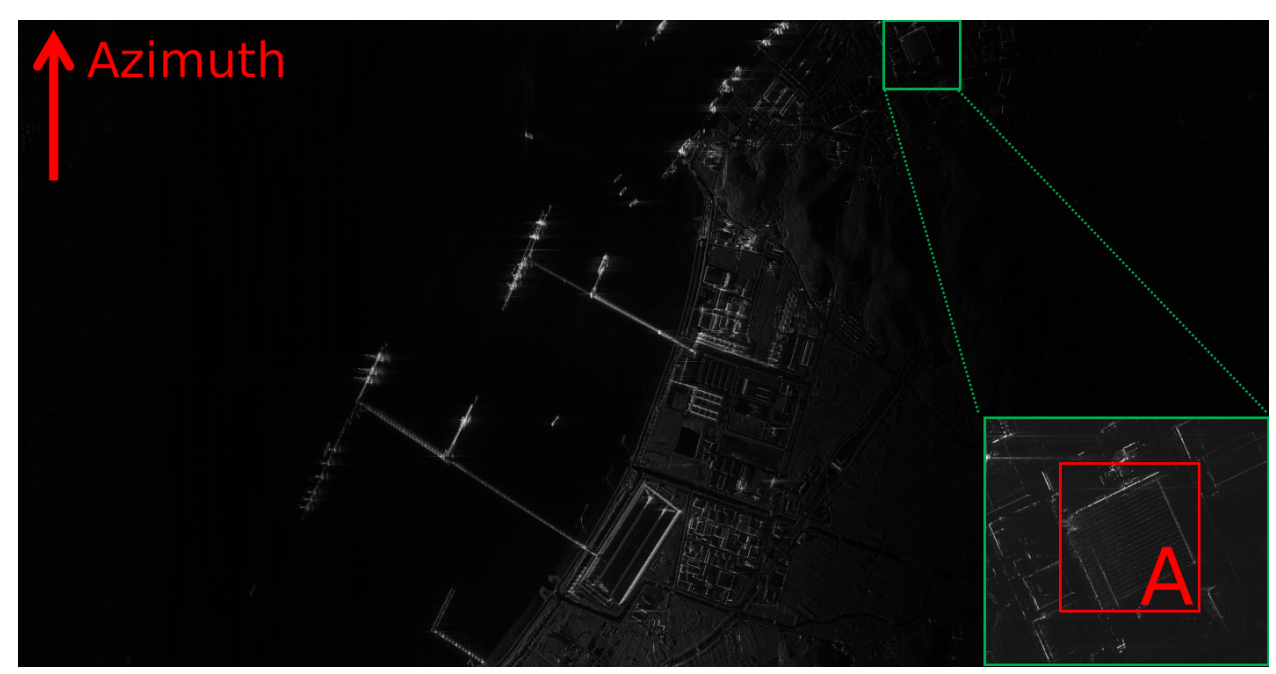

(a)

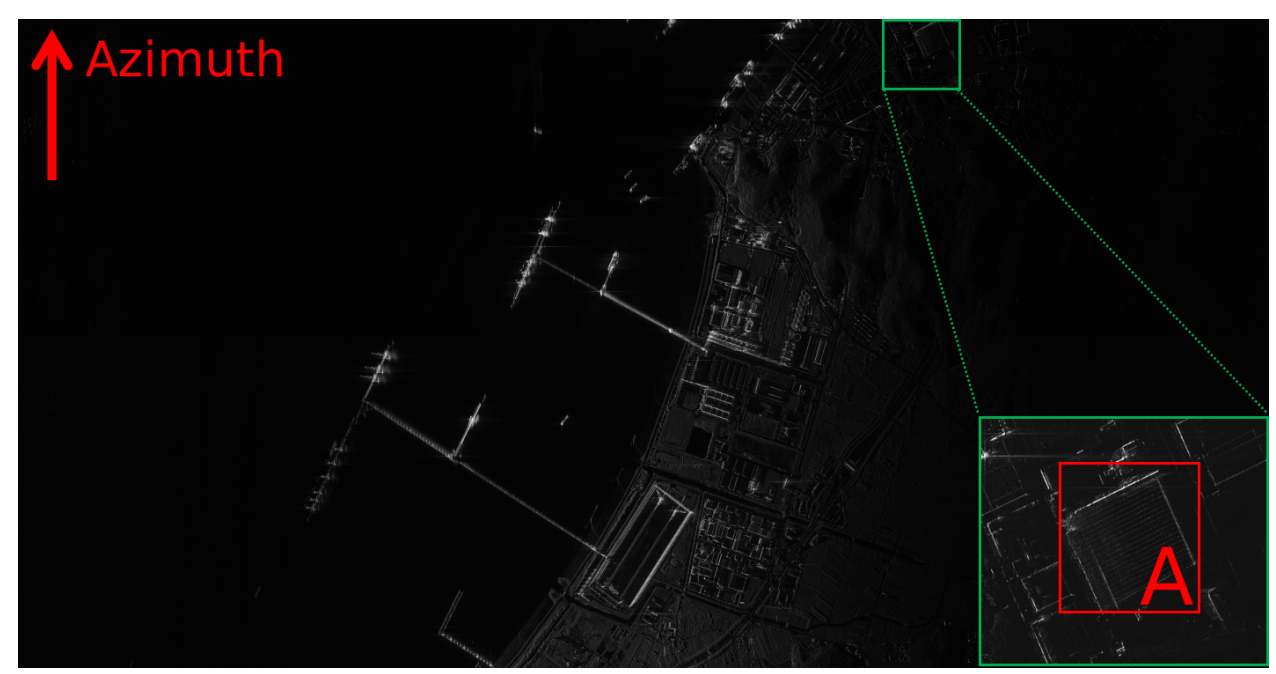

(b)

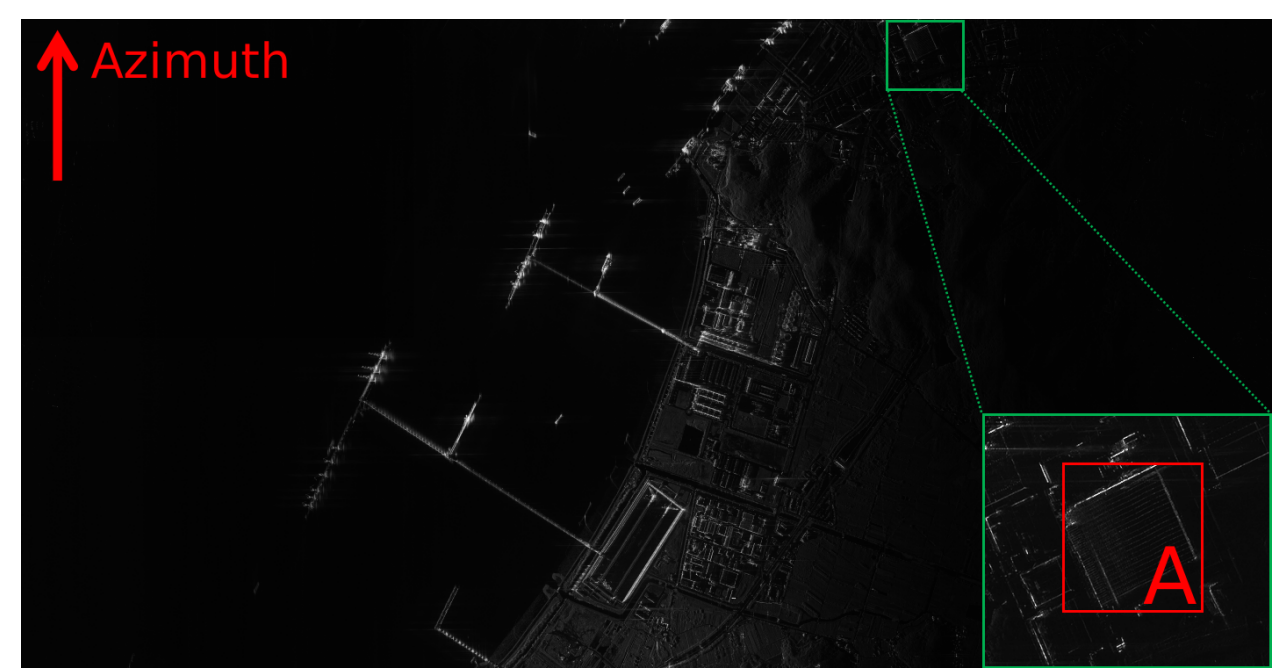

(c)

Figure 17. Experimental results of the 3.6 GHz-bandwidth SAR data. (Provided by the IECAS) (a) Complete image focused with TSM MoCo scheme. (b) Complete image focused with traditional OSM MoCo scheme. (c) Complete image focused with the proposed MoCo scheme. 


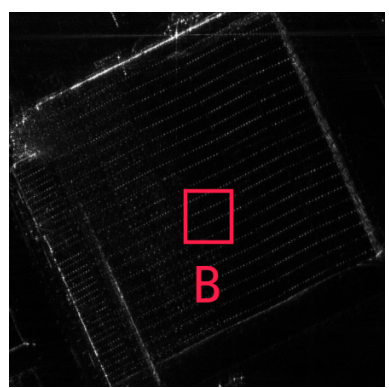

(a)

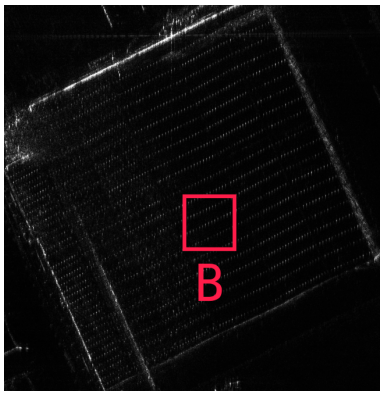

(d)

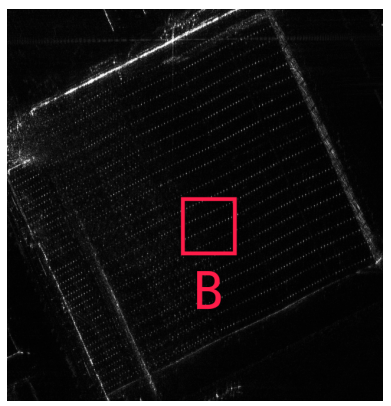

(g)

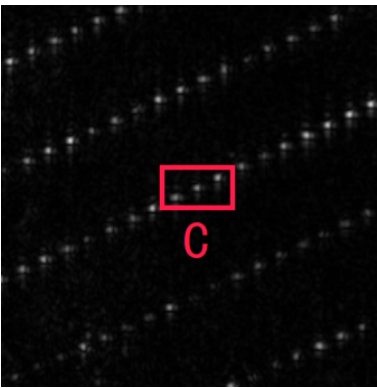

(b)

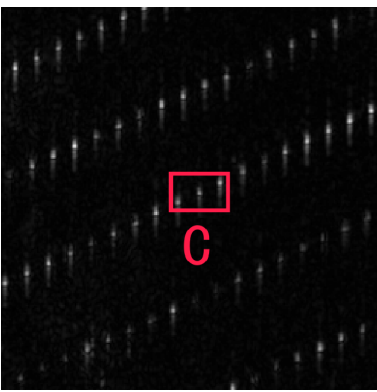

(e)

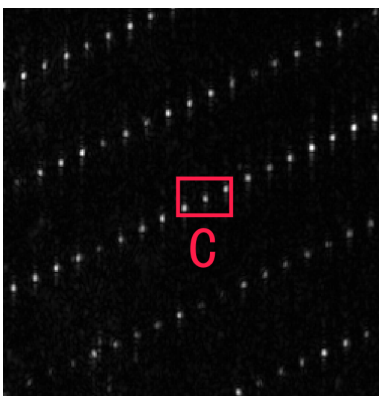

(h)

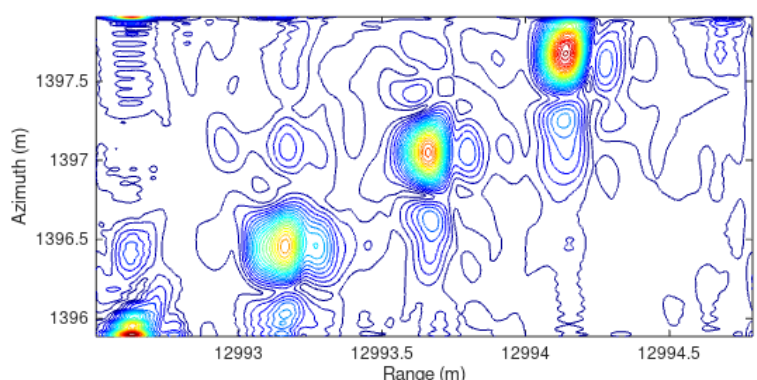

(c)

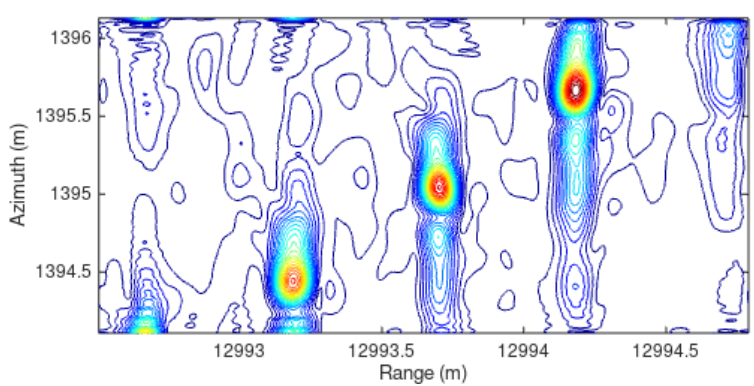

(f)

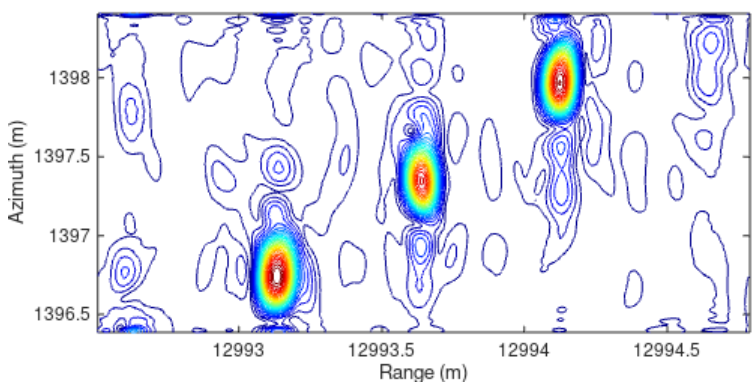

(i)

Figure 18. (a) Area A imaged with TSM MoCo scheme. (b) Area B with TSM MoCo scheme. (c) Area C imaged with TSM MoCo scheme. (d) Area A imaged with traditional OSM MoCo scheme. (e) Area B with traditional OSM MoCo scheme. (f) Area C imaged with traditional OSM MoCo scheme. (g) Area A with the proposed MoCo scheme. (h) Area B imaged with the proposed MoCo scheme. (i) Area $\mathrm{C}$ with the proposed MoCo scheme.

Table 8. Parameters of the Experiment.

\begin{tabular}{cc}
\hline Parameter & Value \\
\hline Carrier frequency & $9.6 \mathrm{GHz}$ \\
Velocity & $126.47 \mathrm{~m} / \mathrm{s}$ \\
Height & $6338 \mathrm{~m}$ \\
Incidence angle & $56.399 \mathrm{deg}$ \\
Signal bandwidth & $3600 \mathrm{MHz}$ \\
Sampling frequency & $4200 \mathrm{MHz}$ \\
Signal pulse duration & $20 \mu \mathrm{s}$ \\
PRF & $3996.2 \mathrm{~Hz}$ \\
Length of antenna & $0.496 \mathrm{~m}$ \\
Height of antenna & $0.32 \mathrm{~m}$ \\
\hline
\end{tabular}




\section{Discussion}

The challenge to high resolution motion compensation is embodied in two aspects. The first aspect is the limited accuracy of the current MoCo method. Traditionally, the LOS displacement correction can be implemented by the bulk compensation in frequency domain. However, for high range resolution or wide range swath SAR, the range-variant motion error is significant. Therefore the method error that ignoring the range-variance displacement will lead to a defocused SAR image. The defocusing effect becomes more obvious as the range offset from the scene center become larger. The selected area Figure 18f,i are selected far from the scene center in the range direction to demonstrate the method error of the traditional MoCo. This challenge can be overcame by updating the MoCo method. As shown in this study, with a more accurate IMU-based MoCo method, the method error of the MoCo can be reduced.

The second aspect of the challenge to high resolution motion compensation is the accuracy of the IMU data. Commonly, the IMU records are precise enough. However, the sampling frequency of IMU is commonly much lower than the PRF. Therefore, it has a time delay for recording the motion error. Although the high-frequency motion error will not be too large for an aircraft, it will accumulate in the whole image since the motion errors for motion compensation are accumulated from the velocities of the IMU record. Besides, the high resolution SAR needs a long aperture time to obtain high azimuth resolution. Therefore, the accuracy of the IMU records degrades with the azimuth swath become longer. Although updating the IMU devices can improve the accuracy, the high cost makes the method become a low performance-to-price ratio scheme. As shown in this study, an alternative method is employing the autofocus methods to estimate the residual motion error and compensate for it. With RCM auto-correction, the residual motion error in range direction can be compensated. Then the PGA can improve the focusing in azimuth direction.

Although the autofocus methods seem to have the ability of compensating the motion error without IMU, it still plays an auxiliary role in the MoCo scheme. The auto-correction has a prerequisite, the high SNR of the signal, for ensuring robustness. With much noise in the signal, it is hard to obtain an accurate estimated results for MoCo. Moreover, the RCM auto-correction will accumulate the estimating error toward azimuth. Therefore, it is necessary to apply some operations, i.e., the frequency filtering, to the low SNR SAR images for enhancing SNR before auto-correction.

The superior limit of the focusing with the proposed MoCo scheme will be lower than imaging and MoCo with the back projection imaging algorithm. The proposed MoCo scheme still has some method errors, i.e., the accuracy of the sub-aperture MoCo. When we divide more sub-apertures, the Doppler frequency of each sub-aperture will be more accuracy while the resolution in time domain of each sub-aperture become more coarser. Besides, the calculating burden will become larger with the increasing number of subapertures. However, when the proposed MoCo scheme can provide the enough accuracy for the MoCo of the given high resolution SAR, the method error can be therefore ignored. The total time cost of the imaging processing in frequency domain and MoCo is much smaller than that of the back projection imaging. Under the circumstances, the proposed MoCo can still be useful.

\section{Conclusions}

In this paper, a novel MoCo scheme is proposed for airborne very high resolution SAR. The proposed MoCo scheme provides more precise range-variant and azimuth-variant compensation for VHR-SAR images compared to traditional methods. Moreover, its performance is verified through both simulations and experimental data from the X-Band $3.6 \mathrm{GHz}$ bandwidth airborne SAR. However, the scheme only considered the zero Doppler centroid mode. For enhancing the flexibility, the MoCo scheme for squint mode will be studied. 
Author Contributions: Conceptualization, Z.C. and Y.Z.; methodology, Z.C.; validation, Z.C. and J.Q.; formal analysis, Z.C.; investigation, Z.Z.; resources, P.W.; data curation, Y.Z.; writing original draft preparation, Z.C. and Y.Z.; writing review and editing, Z.C. and P.W. All authors have read and agreed to the published version of the manuscript.

Funding: This work was funded by the National Natural Science Foundation of China under grant numbers 61971401 and 61901442.

Conflicts of Interest: The authors declare no conflict of interest.

\section{References}

1. Reigber, A.; Alivizatos, E.; Potsis, A.; Moreira, A. Extended wavenumber-domain synthetic aperture radar focusing with integrated motion compensation. IEE Proc. Radar Sonar Navig. 2006, 153, 301-310. [CrossRef]

2. Moreira, A.; Huang, Y. Airborne SAR processing of highly squinted data using a chirp scaling approach with integrated motion compensation. IEEE Trans. Geosci. Remote Sens. 1994, 32, 1029-1040. [CrossRef]

3. Nguyen, M.P. Bewegungskompensation und Autofokussierung von SAR-Rohdaten mit großem Schielwinkel; VDI-Verlag: Duesseldorf, Germany, 2014.

4. Dadi, M.; Donghui, H.U.; Chibiao, D. A New Approach to Airborne High Resolution SAR Motion Compensation for Large Trajectory Deviations. Chin. J. Electron. 2012, 21, 764-769.

5. Yang, M.; Zhu, D.; Song, W. Comparison of two-step and one-step motion compensation algorithms for airborne synthetic aperture radar. Electron. Lett. 2015, 51, 1108-1110. [CrossRef]

6. Ribalta, A. One-step Motion Compensation Algorithm for squinted SAR. In Proceedings of the 2016 IEEE International Geoscience and Remote Sensing Symposium (IGARSS), Beijing, China, 10-15 July 2016; pp. 1154-1157. [CrossRef]

7. Lu, Q.; Gao, Y.; Huang, P.; Liu, X. Range- and Aperture-Dependent Motion Compensation Based on Precise Frequency Division and Chirp Scaling for Synthetic Aperture Radar. IEEE Sens. J. 2019, 19, 1435-1442. [CrossRef]

8. Brunner, D.; Lemoine, G.; Bruzzone, L.; Greidanus, H. Building Height Retrieval From VHR SAR Imagery Based on an Iterative Simulation and Matching Technique. IEEE Trans. Geosci. Remote Sens. 2010, 48, 1487-1504. [CrossRef]

9. Yang, M.; Zhu, D. Efficient Space-Variant Motion Compensation Approach for Ultra-High-Resolution SAR Based on Subswath Processing. IEEE J. Sel. Top. Appl. Earth Observ. Remote Sens. 2018, 11, 2090-2103. [CrossRef]

10. Xiong, T.; Xing, M.; Wang, Y.; Wang, S.; Sheng, J.; Guo, L. Minimum-Entropy-Based Autofocus Algorithm for SAR Data Using Chebyshev Approximation and Method of Series Reversion, and Its Implementation in a Data Processor. IEEE Trans. Geosci. Remote Sens. 2014, 52, 1719-1728. [CrossRef]

11. Li, Y.; Liang, X.; Ding, C.; Zhou, L. Improvements to the Frequency Division-Based Subaperture Algorithm for Motion Compensation in Wide-Beam SAR. IEEE Geosci. Remote Sens. Lett. 2013, 10, 1219-1223.

12. Li, Y.; Liu, C.; Wang, Y.; Wang, Q. A Robust Motion Error Estimation Method Based on Raw Data. IEEE Trans. Geosci. Remote Sens. 2012, 50, 2780-2790. [CrossRef]

13. Yang, L.; Xing, M.; Wang, Y.; Zhang, L.; Bao, Z. Compensation for the NsRCM and Phase Error After Polar Format Resampling for Airborne Spotlight SAR Raw Data of High Resolution. IEEE Geosci. Remote Sens. Lett. 2013, 10, 165-169. [CrossRef]

14. Fornaro, G. Trajectory deviations in airborne SAR: Analysis and compensation. IEEE Trans. Aerospace Electron. Syst. 1999, 35, 997-1009. [CrossRef]

15. Wang, D.; Bai, X.; Zhao, J.; Tao, R. Improved two dimensional autofocus of SAR imaging. In Proceedings of the 20125 th International Congress on Image and Signal Processing, Chongqing, China, 16-18 October 2012; pp. 1822-1826. [CrossRef]

16. Wahl, D.E.; Eichel, P.H.; Ghiglia, D.C.; Jakowatz, C.V. Phase gradient autofocus-a robust tool for high resolution SAR phase correction. IEEE Trans. Aerospace Electron. Syst. 1994, 30, 827-835. [CrossRef]

17. Callow, H.J.; Hayes, M.P.; Gough, P.T. Stripmap phase gradient autofocus. In Proceedings of the Oceans 2003. Celebrating the Past ... Teaming toward the Future (IEEE Cat. No.03CH37492), San Diego, CA, USA, 22-26 September 2003; Volume 5, pp. 2414-2421. [CrossRef]

18. Kantor, J.M. Minimum entropy autofocus correction of residual range cell migration. In Proceedings of the 2017 IEEE Radar Conference (RadarConf), Seattle, WA, USA, 8-12 May 2017; pp. 0011-0016. [CrossRef]

19. Mao, X.; Zhu, D. Two-dimensional Autofocus for Spotlight SAR Polar Format Imagery. IEEE Trans. Comput. Imaging 2016, 2, 524-539. [CrossRef]

20. Mao, X.; Zhu, D.; Zhu, Z. Autofocus Correction of APE and Residual RCM in Spotlight SAR Polar Format Imagery. IEEE Trans. Aerospace Electron. Syst. 2013, 49, 2693-2706. [CrossRef]

21. Mao, X.; Zhu, D.; Zhang, Y.D. Knowledge-aided two-dimensional autofocus for synthetic aperture radar. In Proceedings of the 2013 IEEE Radar Conference (RadarCon13), Ottawa, ON, Canada, 29 April-3 May 2013; pp. 1-6. [CrossRef]

22. Li, J.; Chen, J.; Wang, P.; Loffeld, O. A Coarse-to-Fine Autofocus Approach for Very High-Resolution Airborne Stripmap SAR Imagery. IEEE Trans. Geosci. Remote Sens. 2018, 56, 3814-3829. [CrossRef]

23. Zeng, L.; Liang, Y.; Xing, M.; Li, Z.; Huai, Y. Two-dimensional autofocus technique for high-resolution spotlight synthetic aperture radar. IET Signal Process. 2016, 10, 699-707. [CrossRef] 
24. Li, N.; Wang, R.; Deng, Y.; Yu, W.; Zhang, Z.; Liu, Y. Autofocus Correction of Residual RCM for VHR SAR Sensors with Light-Small Aircraft. IEEE Trans. Geosci. Remote Sens. 2017, 55, 441-452. [CrossRef]

25. Fu, X.; Wang, B.; Xiang, M.; Jiang, S.; Sun, X. Residual RCM Correction for LFM-CW Mini-SAR System Based on Fast-Time Split-Band Signal Interferometry. IEEE Trans. Geosci. Remote Sens. 2019, 57, 4375-4387. [CrossRef]

26. Chen, J.; Liang, B.; Yang, D.; Zhao, D.; Xing, M.; Sun, G. Two-Step Accuracy Improvement of Motion Compensation for Airborne SAR with Ultrahigh Resolution and Wide Swath. IEEE Trans. Geosci. Remote Sens. 2019, 1-13. [CrossRef]

27. Xing, M.; Wu, Y.; Zhang, Y.D.; Sun, G.; Bao, Z. Azimuth Resampling Processing for Highly Squinted Synthetic Aperture Radar Imaging with Several Modes. IEEE Trans. Geosci. Remote Sens. 2014, 52, 4339-4352. [CrossRef]

28. Prats, P.; Camara de Macedo, K.A.; Reigber, A.; Scheiber, R.; Mallorqui, J.J. Comparison of Topography- and Aperture-Dependent Motion Compensation Algorithms for Airborne SAR. IEEE Geosci. Remote Sens. Lett. 2007, 4, 349-353. [CrossRef]

29. Zheng, X.; Yu, W.; Li, Z. A Novel Algorithm for Wide Beam SAR Motion Compensation Based on Frequency Division. In Proceedings of the 2006 IEEE International Symposium on Geoscience and Remote Sensing, Denver, CO, USA, 31 July-4 August 2006; pp. 3160-3163. [CrossRef]

30. Zhou, Y.; Wang, P.; Ye, K.; Deng, Y.; Wang, R.; Zhang, H.; Zhao, Q. A 3.6 GHZ X-Band Wideband Experimental Airborne Sar System. In Proceedings of the IGARSS 2019, 2019 IEEE International Geoscience and Remote Sensing Symposium, Yokohama, Japan, 28 July-2 August 2019; pp. 8629-8632. 